\title{
Uterine Rbpj is required for embryonic-uterine orientation and decidual remodeling via Notch pathway-independent and -dependent mechanisms
}

Shuang Zhang ${ }^{1, *}$, Shuangbo Kong, 2, *, Bingyan Wang ${ }^{1}$, Xiaohong Cheng ${ }^{1,3}$, Yongjie Chen ${ }^{1,2}$, Weiwei Wu ${ }^{1,2}$, Qiang Wang ${ }^{1}$, Junchao Shi ${ }^{1,2}$, Ying Zhang ${ }^{1}$, Shumin Wang ${ }^{1}$, Jinhua Lu ${ }^{1}$, John P Lydon ${ }^{4}$, Francesco DeMayo ${ }^{4}$, Warren S Pear ${ }^{5}$, Hua Han ${ }^{6,7}$, Haiyan Lin ${ }^{1}$, Lei Li ${ }^{1}$, Hongmei Wang ${ }^{1}$, Yan-ling Wang ${ }^{1}$, Bing Li ${ }^{3}$, Qi Chen ${ }^{1}$, Enkui Duan ${ }^{1}$, Haibin Wang ${ }^{1}$

${ }^{I}$ State Key Laboratory of Reproductive Biology, Institute of Zoology, Chinese Academy of Sciences, Beijing 100101, China; ${ }^{2}$ University of Chinese Academy of Sciences, Beijing 100101, China; ${ }^{3}$ Reproductive Medical Center, The Third Affiliated Hospital of Guangzhou Medical College, Key Laboratory for Major Obstetric Diseases of Guangdong Province, Guangzhou, Guangdong, China; ${ }^{4}$ Department of Molecular and Cellular Biology, Baylor College of Medicine, Houston, TX 77030, USA; ${ }^{5}$ Department of Pathology, Perelman School of Medicine, University of Pennsylvania, Philadelphia, PA 19104, USA; ${ }^{6}$ Department of Dermatology, Xijing Hospital, ${ }^{7}$ State Key Laboratory of Cancer Biology, Department of Medical Genetics and Developmental Biology, Fourth Military Medical University, Xi'an, Shanxi 710032, China

Coordinated uterine-embryonic axis formation and decidual remodeling are hallmarks of mammalian post-implantation embryo development. Embryonic-uterine orientation is determined at initial implantation and synchronized with decidual development. However, the molecular mechanisms controlling these events remain elusive despite its discovery a long time ago. In the present study, we found that uterine-specific deletion of Rbpj, the nuclear transducer of Notch signaling, resulted in abnormal embryonic-uterine orientation and decidual patterning at post-implantation stages, leading to substantial embryo loss. We further revealed that prior to embryo attachment, Rbpj confers on-time uterine lumen shape transformation via physically interacting with uterine estrogen receptor $(E R \alpha)$ in a Notch pathway-independent manner, which is essential for the initial establishment of embryo orientation in alignment with uterine axis. While at post-implantation stages, Rbpj directly regulates the expression of uterine matrix metalloproteinase in a Notch pathway-dependent manner, which is required for normal post-implantation decidual remodeling. These results demonstrate that uterine $\mathbf{R b p j}$ is essential for normal embryo development via instructing the initial embryonic-uterine orientation and ensuring normal decidual patterning in a stage-specific manner. Our data also substantiate the concept that normal mammalian embryonic-uterine orientation requires proper guidance from developmentally controlled uterine signaling.

Keywords: Rbpj; embryo orientation; decidual remodeling; ER $\alpha$; MMPs; DNMAML

Cell Research (2014) 24:925-942. doi:10.1038/cr.2014.82; published online 27 June 2014

*These two authors contributed equally to this work.

Correspondence: Haibin Wang ${ }^{\mathrm{a}}$, Enkui Duan ${ }^{\mathrm{b}}$, Qi Chen ${ }^{\mathrm{c}}$

${ }^{a}$ Tel: 86-01-64807868

E-mail: hbwang@ioz.ac.cn

${ }^{\mathrm{b}, \mathrm{c}}$ Tel: 86-01-64807182

bE-mail: duane@ioz.ac.cn

${ }^{c} E-m a i l:$ chenqi@ioz.ac.cn

Received 27 January 2014; revised 14 April 2014; accepted 13 May 2014;

published online 27 June 2014

\section{Introduction}

During implantation, rodent blastocysts show remarkably consistent embryonic orientation with respect to the uterine environment; the entire blastocyst is located at the antimesometrial pole of the uterine vertical axis, while the inner cell mass (ICM) is positioned at the mesometrial side of the implantation chamber [13]. Simultaneously, the ICM-abembryonic axis is nearly coincident with the uterine mesometrial-antimesometrial 
(M-AM) axis in the vertical direction [4]. The embryonic orientation at the time of initial implantation directs the development of future uterus-embryo alignment during post-implantation stages [5], which is synchronized with the uterine shape transformation that is driven by patterned stromal decidualization [6]. However, despite the morphological implications, the precise molecular mechanisms that guide proper embryonic orientation and the signaling pathways by which uterine-embryonic axis progression is coordinated with the uterine decidualization remain largely unexplored $[4,7,8]$.

Structurally, the uterine lining at the time of initial embryo implantation intimately interacts with the blastocyst, providing the opportunity for an instructive role in the blastocyst development, especially regarding the positional and orientation clues that would blueprint the subsequent uterine-embryonic axis formation [8-10]. Before the initial uterine-embryo physical attachment, the endometrium undergoes substantial changes under the coordination of ovarian estrogen and progesterone. The overall shape of the uterine lumen transforms from initially containing many randomly formed epithelial folds, to a highly regulated slit-like structure, with its long axis in a M-AM direction; this lumen shape transformation process is usually termed "luminal closure" $[4,11,12]$. Since the axis of the lumen is in alignment with future embryonic axis, we have recently proposed that the ontime luminal closure is a critical factor that would guide and facilitate the correct embryonic orientation at initial implantation [8]. If the luminal closure could not happen normally, the embryo would lose positional clues in the uterine lumen and fail to establish the correct initial embryo orientation at implantation. The differential decidual remodeling and vasculature development within the stromal bed at post-implantation stages may also play an active role in the final establishment of uterine boundary and thus essential for uterine-embryonic axis optimization and embryo survival $[13,14]$. We hypothesized that developmentally controlled uterine gene expression during the peri-implantation period may play essential roles in guiding these events.

The Notch signaling pathway is highly evolutionarily conserved and has been demonstrated to play diverse roles in multiple cellular and organ functions. Notch signaling is initiated by the interaction between the Notch receptor (Notch 1, 2, 3 and 4) and its ligand, leading to the release of the Notch intra-cellular domain (NICD), which translocates to the nucleus and interacts directly with Rbpsuh (Rbpj), together with Mastermind-like protein (MAML) to exert transcriptional activity [15, 16]. Rbpj could also function independently of Notch signaling and form complexes with other transcription factors $[17,18]$. A wealth of data have demonstrated the importance of Rbpj in stem cell maintenance, cell fate decisions, cell proliferation, differentiation, and embryo patterning such as dorsal-ventral axis establishment and left-right asymmetry determination [19-23]. Systemic Rbpj deletion in mice results in embryonic lethality prior to 10.5 days of gestation due to the accumulation of multiple abnormalities, such as growth retardation, defective somitogenesis, and abnormal placental development [24]. However, it is unclear whether uterine Rbpj play any roles in peri-implantation events and post-implantation embryo-uterus interactions.

In the current study, by using a uterine-specific deletion mouse model, we provided novel evidence that although on-time embryo implantation occurs in the absence of uterine Rbpj, the normal embryonic orientation is disrupted at the time of initial implantation and results in a progressive uterine-embryonic disorientation associated with aberrant decidual patterning. We further revealed that Rbpj influences the initial embryonic orientation by regulating on-time uterine lumen transformation (luminal closure), through physical interaction with and suppression of the overactive function of uterine estrogen receptor $\alpha(E R \alpha)$ in a Notch pathway-independent manner. At post-implantation stages, Rbpj directly regulates the expression of matrix metalloproteinase (MMP) genes in a Notch pathway-dependent manner, which is essential for decidual remodeling. These data demonstrate the critical roles of uterine Rbpj in directing peri-implantation uterine transformation and thus embryonic development, reinforcing a concept that developmentally regulated uterine signaling is required to establish proper uterine-embryonic orientation and decidual patterning that are essential for embryo development.

\section{Results}

\section{Rbpj is spatiotemporally expressed in peri-implantation mouse uteri}

In our attempts to screen potential candidate genes involved in peri-implantation uterine transformation, we found that the Notch signaling effector, Rbpj, is spatiotemporally expressed in the peri-implantation mouse uteri as revealed by in situ hybridization analysis (Figure $1 \mathrm{~A})$. On day 1 of pregnancy, $R b p j$ was weakly expressed in the luminal epithelial cells; however, on day 4 (prior to embryo attachment), Rbpj localization shifted to the sub-epithelial uterine stroma region. With the onset of implantation on day 5 , the expression expanded to the entire stromal bed. Accompanied by the progression of embryo implantation, Rbpj was intensely expressed in the decidualizing cells on the antimesometrial half of 
A
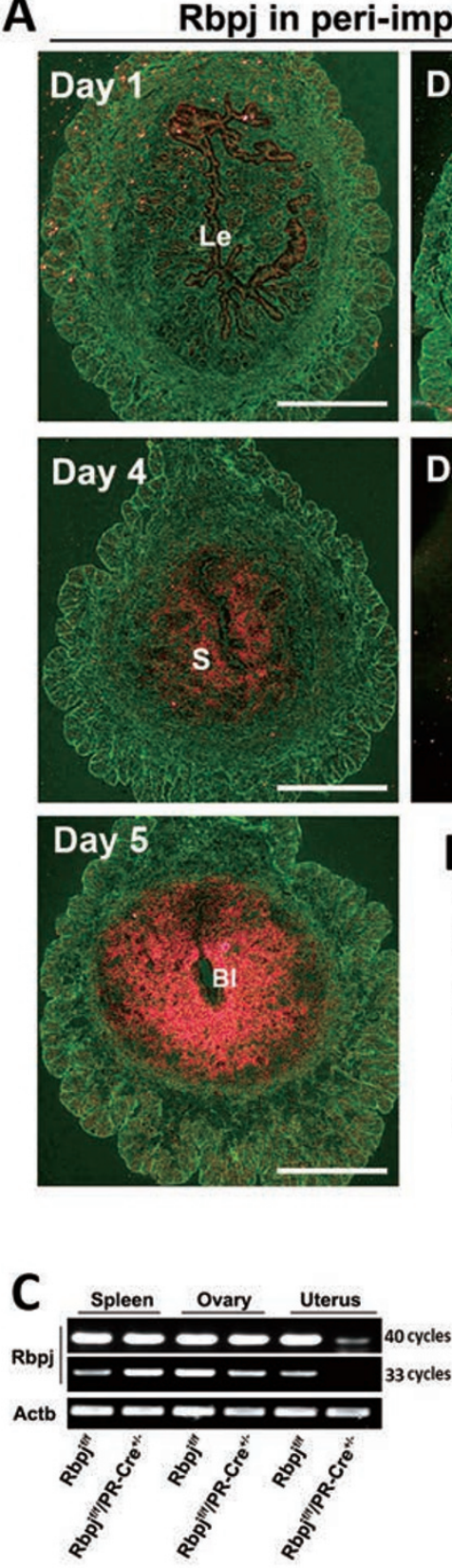

E
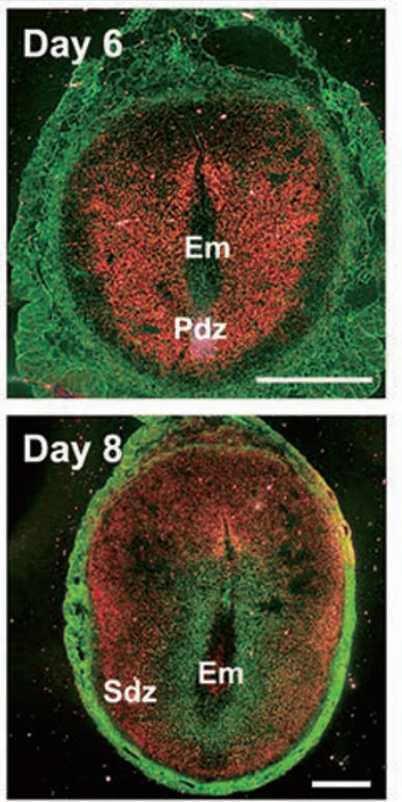

B
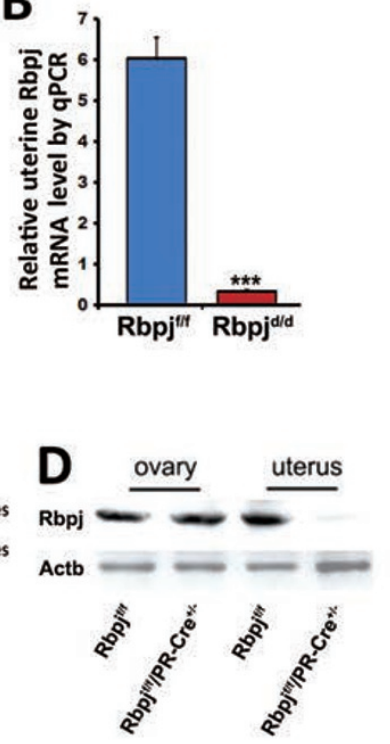
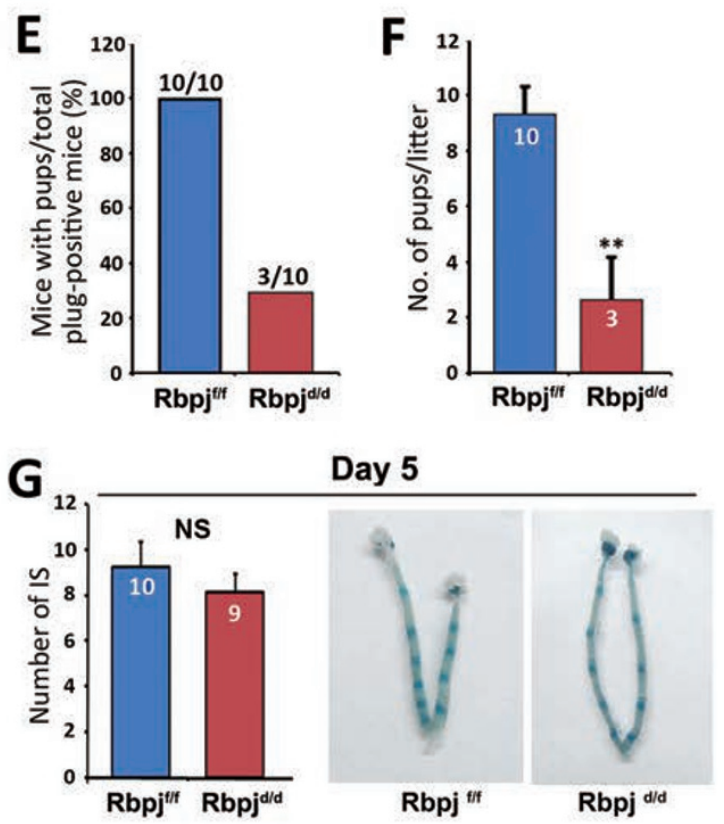

H

Day 8

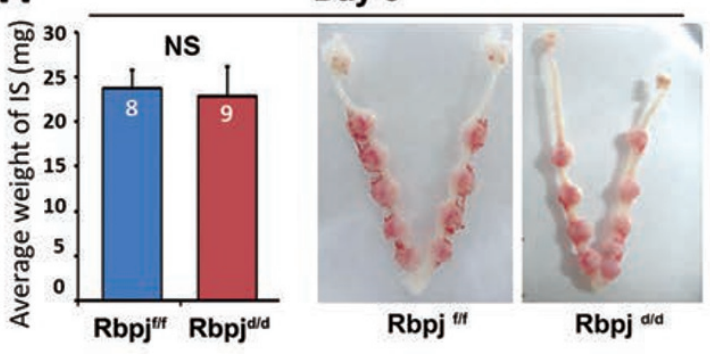

Day 12

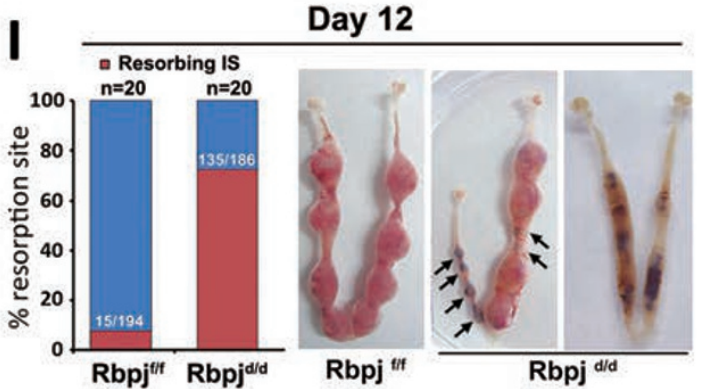

Figure $1 \mathrm{Rbpj}$ is expressed in a spatiotemporal manner in the uterus and is critical for normal pregnancy. (A) In situ hybridization showing the spatiotemporal expression of $R b p j$ in WT uteri on days 1, 4, 5, 6 and 8 of pregnancy. BI, blastocyst; Le, luminal epithelium; Pdz, primary decidual zone; Sdz, secondary decidual zone; S, stroma. (B) Real-time quantitative PCR analysis of uterine $R b p j$ mRNA in $R b p f^{f / f}$ and $R b p j j^{d / d}$ mice. Data shown represent the mean \pm SEM. $* * * P<0.01$. (C) RT-PCR analysis of $R b p j$ expression in the spleen, ovary and uterine stromal cells of $R b p j j^{f / f}$ and $R b p j / d$ mice. $R b p j$ mRNA expression was efficiently deleted in the uteri of the $R b p j^{d / d}$ mice but still abundant in the spleen and ovary. (D) Immunoblotting analysis of Rbpj protein in the ovaries and uteri on day 4 of pregnancy dissected from $R b p j^{f / f}$ and $R b p j^{i / d}$ mice. (E) Pregnancy outcomes in $R b p j^{f / f}$ and $R b p j^{d / d}$ mice. (F) Average litter sizes in $R b p j^{f / f}$ and $R b p j^{d / d}$ mice. ${ }^{*} * P<0.01$. (G) Morphologically normal implantation in $R b p j^{d / d}$ mice compared with $R b p j^{f / f}$ mice as determined by blue dye injection on day 5 . The average number of implantation sites is comparable between the $R b p j^{f / f}$ and $R b p j^{d / d}$ mice. IS, implantation site; NS, not significant. (H) The weight of the implantation sites and representative uteri from $R b p j^{f / f}$ and $R b p j^{i / d}$ females on day 8 of implantation. IS, implantation site; NS, not significant. (I) Resorption rate and representative uteri from $R b p j^{f / f}$ and $R b p j j^{d / d}$ females on day 12 . The black arrowheads denote the resorption sites. In I, the numbers within bars indicate the number of resorption events divided by the total number of implantation sites. In F-H, the numbers within bars indicate number of females examined for each group. 
day 6 uteri, while the expression shifted to the secondary decidual zone and the mesometrial portion on day 8 (Figure 1A). We also found that the expression pattern of Notch ligands and receptors in peri-implantation uteri is overlapping with that of Rbpj in a spatiotemporal manner (Supplementary information, Figure S1). Given the central role of Rbpj in Notch signaling, the dynamic uterine expression pattern of Rbpj led us to study its potential roles in regulating both pre- and post-implantation uterine functions.

Uterine-specific Rbpj deletion results in embryo resorption and miscarriage at mid-gestation

To examine the potential functions of Rbpj in mouse uteri, we generated a mouse model harboring a uterine-specific deletion of $R b p j\left(R b p j{ }^{d / d}\right)$ by crossing $\mathrm{Rb}$ pj-loxp mice $\left(\mathrm{Rbpj}^{f / f}\right)$ with $P R$-Cre mice $\left(P R-\mathrm{Cre}^{+/}\right)[25-$ 27]. $P R$-Cre-mediated recombination in uterus is of high efficiency (Supplementary information, Figure S2). In $\mathrm{Rbpj}^{d / d}$ mice, Rbpj expression in uteri was specifically abolished at both the mRNA and protein levels, while its expression in other tissues remained unaffected (Figure 1B-1D). To test the role of Rbpj in female fertility, $R b p j^{f / f}$ and $R b p j^{d / d}$ females were mated with wild-type (WT) males. Females of both genotypes mated normally and formed vaginal plugs. However, the pregnancy rate and litter size were markedly lower in the $R b p j^{d / d}$ females compared with the $R b p j^{f / f}$ females (Figure $1 \mathrm{E}$ and $1 \mathrm{~F}$ ), demonstrating that uterine Rbpj is crucial for normal female fertility.

To identify the specific stage by which the pregnancy defects occurred, we examined the pregnancy status on days 5, 8 and 12, which represent the timing of embryo implantation, decidualization, and mid-gestation, respectively. In the $R b p j^{d / d}$ mice, while the pregnant uteri seemed normal on days 5 and 8 as indexed by the gross number and weight of the implantation sites, respectively (Figure $1 \mathrm{G}$ and $1 \mathrm{H}$ ), clear defects were observed on day 12 of pregnancy, showing that a substantial number of implantation sites had already been completely absorbed (Figure 1I). These data demonstrate that the major pregnancy defects leading to embryonic lethality may have originated around the time of post-implantation to mid-gestation.

Uterine-embryonic disorientation and abnormal decidual patterning lead to defective embryo development in Rbpj mutant mice

To elucidate the underlying causes of the observed pregnancy loss in $R b p j^{d / d}$ mice, we next carefully examined the post-implantation events from day 6 to day 8 . The data showed that the gross morphology and weight of implantation sites were comparable in $\mathrm{Rbpj}^{\mathrm{d} / \mathrm{d}}$ and $R \mathrm{R}$ $p j^{f / f}$ females (Figure $1 \mathrm{G}$ and $1 \mathrm{H}$ ). However, upon closer investigation, the histological appearance of the tissue sections revealed a post-implantation phenotype with a substantial number $(\sim 35 \%)$ of $R b p j^{d / d}$ implantation sites harboring a severely deflected uterine-embryonic axis ( $>10^{\circ}$ angle) from day 6 to day 8) (Figure 2A-2D), suggesting that this defect has an even earlier developmental origin.

In addition to the deflected uterine-embryonic axis in the $\mathrm{Rbpj}^{d / d}$ females, we also observed an aberrant uterine boundary and decidual shape characterized by a shortened uterine long/short axis ratio; this phenotype was not obvious on day 6 , but it became progressively exaggerated with the decidualization progression beyond day 7 (Figure 2A and 2E), suggesting that these defects are interdependent in the post-implantation uterine decidualization process. Moreover, these changes in decidual pattern are also well reflected in the expression patterns of several decidual marker genes such as $I L-11 R a$, Wnt4 and Bmp2 [28-31]. As shown in Supplementary information, Figure S3, while the intensity of these genes may not change significantly, the regional expression pattern has become apparently abnormal due to the defects in uterine architecture.

These embryonic orientation and decidual pattern defects also caused severe abnormal embryonic development characterized by a significantly reduced embryonic length and area (Figure $2 \mathrm{~F}-2 \mathrm{H}$ ), indicating restrained embryonic growth. This defect might be in part due to abnormal mechanical forces caused by changed embryonic orientation in alignment with uterine architecture, limiting the embryo to "stretch out". Moreover, by examining the anterior visceral endoderm (AVE) marker Cerl1 in both tissue sections and isolated embryos, which would indicate the progression of embryonic anterior-posterior (AP) axis establishment $[32,33]$, we found that although the embryos in mutant uteri express Cerl1 and some positive cells seem to have localized to the right location as compared to the asymmetrical expression in normal mice, the overall development of embryo is retarded and the shape of the embryo is apparently constrained (Figure 2I), which are most possibly accounting for the subsequent embryonic lethality.

Uterine-embryonic disorientation is caused by deflected embryonic axis established within abnormal uterine crypts, due to defective luminal closure

To further elucidate the origin of the defective embryo orientation at post-implantation stages, we performed detailed histological examinations coupled with immunofluorescence staining of E-cadherin or cytokeratin 
A

A
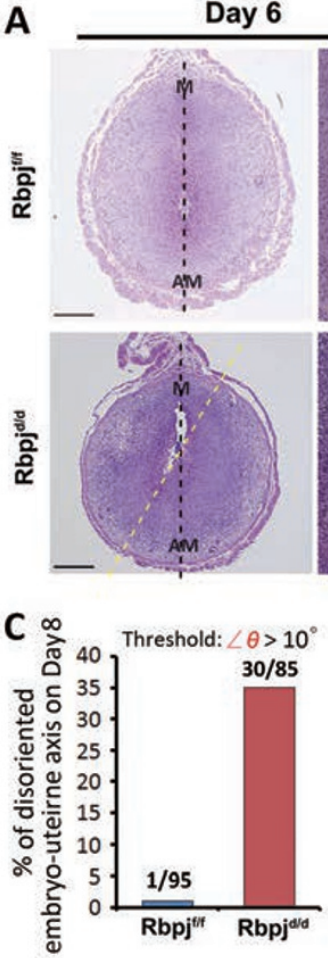

$\mathbf{F}$

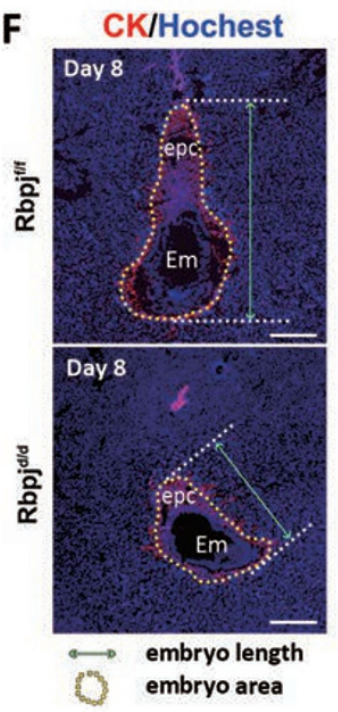

Day 8
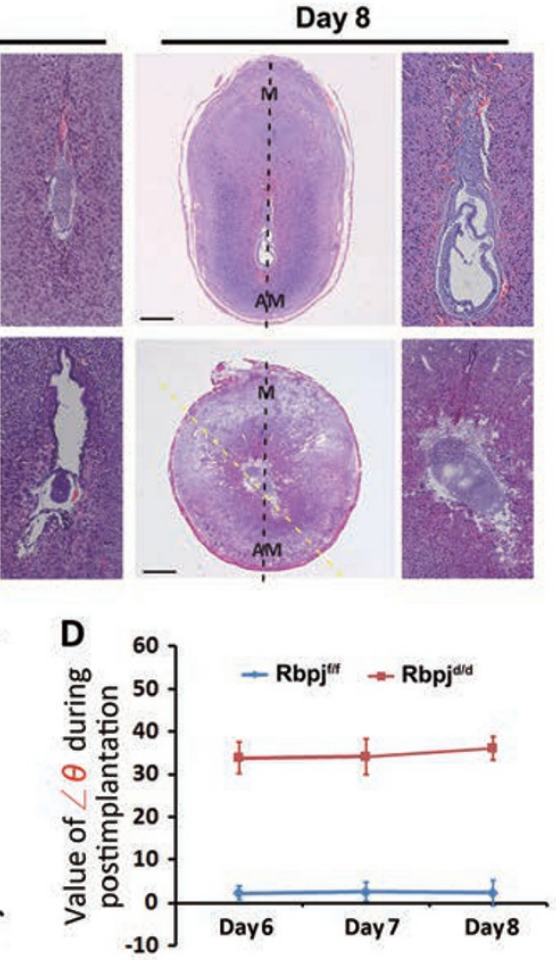

B

. . - : Uterine vertical axis (M-AM axis)

-.- : Embryonic axis

$\angle \theta$ : Angle of uteirne and embryonic axis

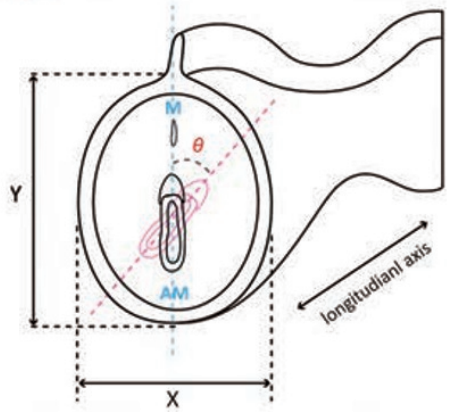

$\mathrm{Y} / \mathrm{X}$ : Ratio of uterine long/short axis

E

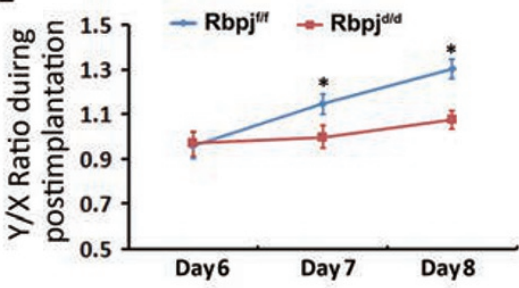

G
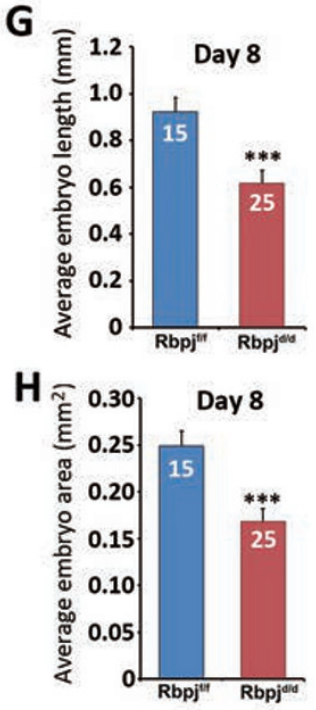

I
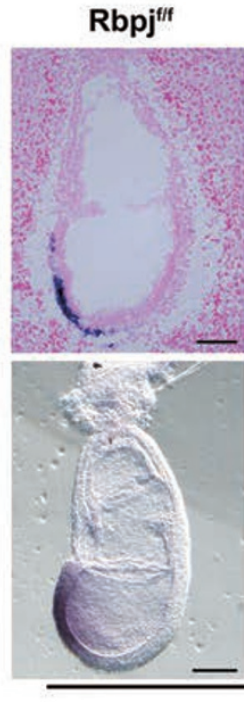

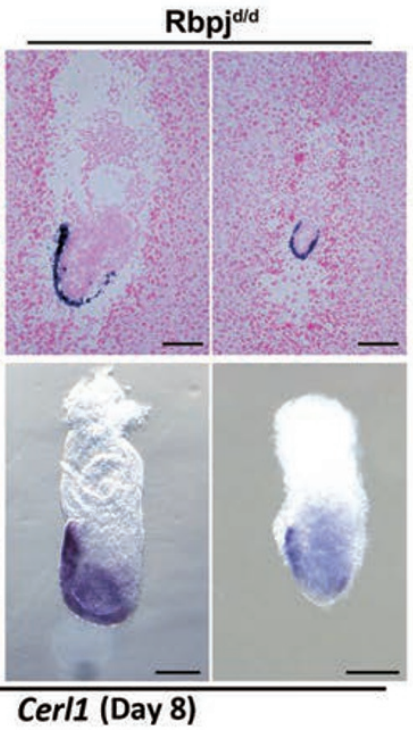

Figure 2 Aberrant embryonic-uterine orientation and decidual patterning in $R b p j^{d / d}$ females. (A) Representative cross-sections of $R b p j^{f / f}$ and $R b p j^{d / d}$ implantation sites on days 6 and 8 . The narrow columns show a magnified embryonic image of the left pictures. The black dashed line shows the uterine long axis, and the yellow dashed line shows the embryonic long axis. AM, antimesometrial pole; M, mesometrial pole. Scale bar, $500 \mu \mathrm{m}$. (B) Schematic diagram illustrating the embryonic orientation within the uterus and the relative position of the section plane. The embryonic axis is parallel to the uterine M-AM axis in the $R b p j^{f / f}$ uteri, while the embryonic axis (delineated by the red dashed line) is deflected from the uterine axis in the $R b p j^{-d / d}$ uteri. The angles of deviation are indicated as $\angle \theta$. (C) The percentage of uterine-embryo disorientation on day 8 for $R b p j^{f / f}$ and $R b p j^{d / d}$ implantation sites. The threshold of $\angle \theta$ analyzed is $>10^{\circ}$. (D) The value of $\angle \theta$ during post-implantation stages in $R b p j^{f / f}$ and $R b p j^{d / d}$ implantation sites. $n=80-90$ implantation sites for each time point. (E) The ratio of the uterine short (X) and long (Y) axes during days $6-8$ in the $R b p j^{f / f}$ and $R b p j^{d / d}$ implantation sites, $n=80-90$ implantation sites for each time point. Data shown represent the mean \pm SEM. $* P<0.05$. (F) Illustration for measurements of embryo length and embryo area (including ectoplacental cone (epc)) in $R b p j^{f / f}$ and $R b p j^{d / d}$ implantation sites. (G, H) Average embryo length (G) and embryo area (H) in $R b p j^{f / f}$ and $R b p j^{* / d}$ implantation sites. Data shown represent the mean \pm SEM. $* * * P<0.01$. (I) In situ hybridization of Cerl1 in day 8 implantation sites and embryos isolated from $R b p j^{f / f}$ and $R b p j^{i / d}$ mice. 
(epithelial markers) at the pre-implantation and initial implantation stages. As illustrated in Figure 3A and 3B, the day 3 and day 4 pregnant uteri showed normal luminal closure in $R b p j^{f / f}$ mice; the luminal structure changed from a randomly folded shape to a highly regulated slitlike structure with the long axis parallel to the uterine M-AM direction. However, the $R b_{p j} j^{d / d}$ uteri showed incomplete luminal closure on day 4 , which was indicated by the epithelial folds that remained at both the mesometrial and antimesometrial sides (Figure 3A and 3B). These abnormally formed epithelial folds, therefore, served as many "pitfalls" for the blastocyst at the time of initial implantation. If a blastocyst implants in an abnormal epithelial fold and then establishes its embryo orientation (deflected with respect to the M-AM axis), this slight deflection at initial implantation will lead to a progressively disoriented uterine-embryonic axis at post-implantation stages. As shown in Figure 3C and Supple-

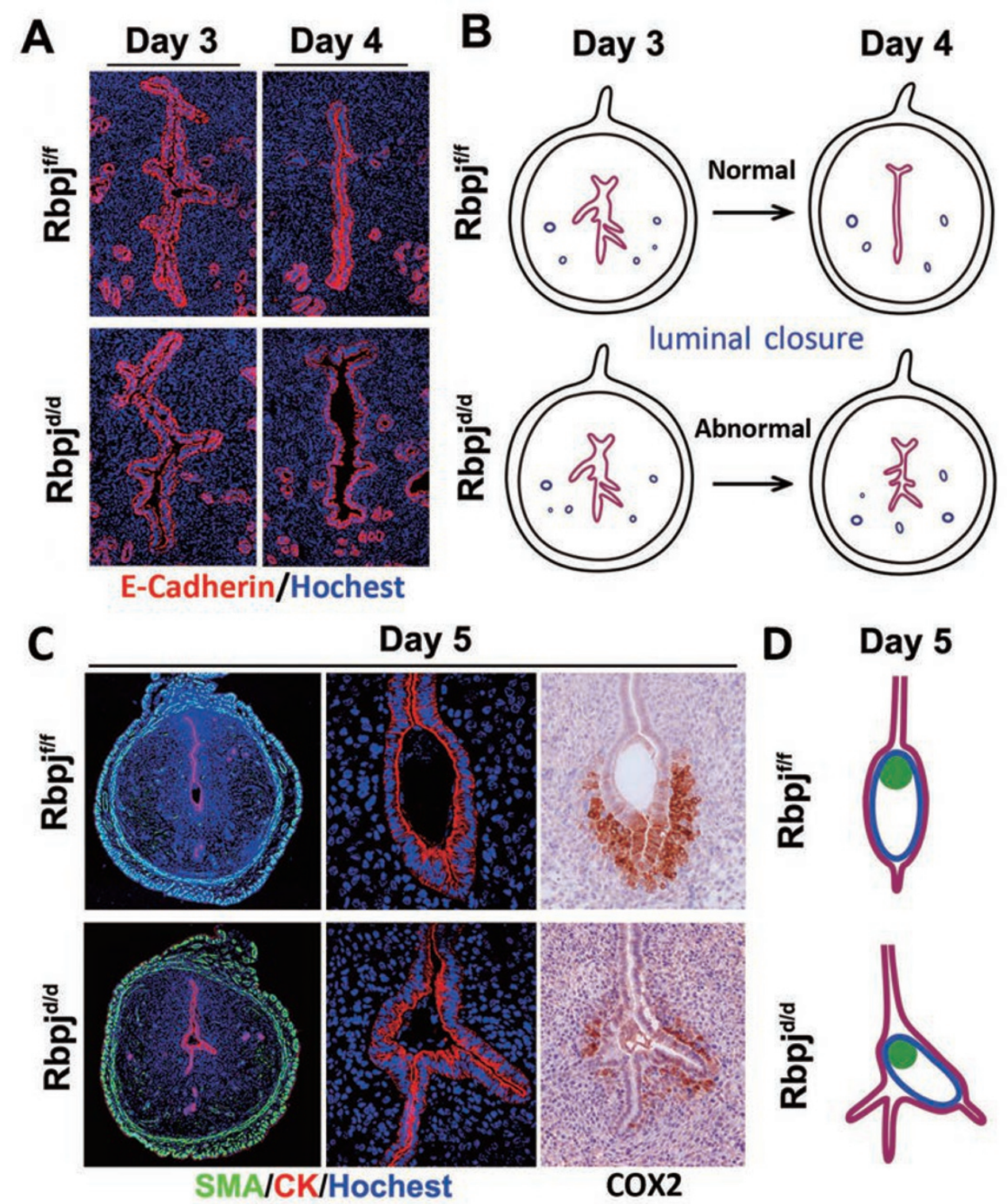

Figure $3 \mathrm{Rbpj}^{\mathrm{d} / \mathrm{d}}$ females show defective luminal closure and deflected embryo orientation at the time of initial implantation. (A) Abnormal luminal closure and increased epithelial branching in $R b p j^{d / d}$ females evidenced by the immunofluorescence of E-cadherin in day 3 and day 4 uteri. Cy3-labeled E-cadherin is shown in red; Hoechst 33342-labeled nuclei are shown in blue. (B) Diagram illustrating the process of luminal closure from day 3 to day 4 in $R b p j^{f / f}$ and $R b p j^{d / d}$ uteri. (C) Co-immunofluorescence of CK and SMA and the immunohistochemistry of COX2 in $R b p j^{f / f}$ and $R b p j^{d / d}$ implantation sites on day 5. SMA, smooth muscle actin; CK, cytokeratin. (D) Diagram of embryo implantation in day 5 implantation sites in $R b p j^{f / f}$ and $R b p j^{i / d}$ mice. 
mentary information, Figure S4, and delineated in Figure $3 \mathrm{D}$, the implanting blastocysts in the $R b p j^{d / d}$ uteri used an abnormal epithelial fold as the implantation chamber. The embryonic disorientation was further demonstrated by the expression of $\mathrm{COX} 2$, an implantation marker that is typically localized around the antimesometrial side of the normal implantation chamber $[34,35]$, which is abnormally located along with the deflected embryo orientation in $\mathrm{Rbpj}^{d / d}$ mice (Figure 3C).

Notably, in some implantation sites, while there are also branched crypts, the embryo ended up in the relatively normal crypt and established an axis that is similar to the normal condition (Supplementary information, Figure S5). Such situation could be explained as a chance event that the embryo could end up into any one of the several potential crypts in the Rbpj mutant uteri (while in normal uteri, there is only one crypt with its axis parallel to uterine M-AM direction), establishing an embryonic axis that is dependent on the direction of the crypt that it accommodates with.

Collectively, these results indicate that the observed uterine-embryonic disorientation is originated from abnormal luminal closure prior to embryonic attachment, and subsequent deflected embryonic axis in alignment with abnormal uterine crypts.

Defective luminal closure in Rbpj ${ }^{d / d}$ uteri is associated with enhanced uterine estrogen response

The process of luminal closure is regulated by a progesterone $\left(\mathrm{P}_{4}\right)$-primed uterine environment in mice. Since the ovary is the primary source of plasma $\mathrm{P}_{4}$ at this stage, we examined ovarian histology, the expression of $\mathrm{P}_{4}$-producing enzymes, and the serum $\mathrm{P}_{4}$ level in both $R_{b p j} j^{d / d}$ and $R b p j^{f / f}$ mice. As shown in Supplementary information, Figure S6A and S6B, the ovarian histology, $\mathrm{P}_{4}$ levels, and the expression of the $\mathrm{P}_{4}$ biosynthetic enzymes cytochrome P450 cholesterol side-chain cleavage enzyme (P450scc) and 3 $\beta$-hydroxysteroid dehydrogenase

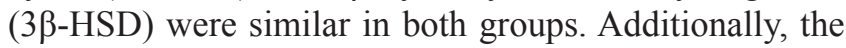
expression of the progesterone receptor (PR) and the $\mathrm{P}_{4}$ target genes Indian hedgehog $(\mathrm{Ihh})$ and amphiregulin (Areg) [26, 36] was also comparable (Supplementary information, Figure S6C and S6D), suggesting that Rbpj deficiency has no adverse effects on $\mathrm{P}_{4}$ function in the null uterus.

As $\mathrm{P}_{4}$ exerts its effects both synergistically and antagonistically with estrogen through their receptors in the uterus [12], we hypothesized that the abnormal luminal closure in the $\mathrm{Rbpj}^{d / d}$ uteri may have resulted from an abnormal estrogenic response. To test this hypothesis, we first examined the expression of stromal estrogen-responsive genes. As shown in Figure 4A, the levels of Igfl,
$F g f 2,8$, and $18[37,38]$ on day 4 were significantly upregulated in the $R b p j^{d / d}$ mice compared with the $R b p j^{f f f}$ mice. Interestingly, the levels of Lactoferrin (Ltf) and Mucin-1 (Muc1), which are estrogen-sensitive markers in the uterine epithelium [39], were also significantly upregulated (Figure 4A). The enhanced uterine estrogen response was further evidenced by the increased epithelial proliferation demonstrated by Ki67 staining and the aberrant expression of the phospho-estrogen receptor $\alpha(p-E R \alpha)$ (Figure 4B), while the serum level of $17 \beta$-estradiol $\left(\mathrm{E}_{2}\right)$ and the expression of ER $\alpha$ did not show obvious changes (Supplementary information, Figure S6A and S6D). Further analysis of luminal epithelial structure by light and electron microscopy revealed that the $R b p j^{d / d}$ epithelial surface failed to undergo membrane flattening on day 4 (Figure 4C), suggesting an impaired epithelial transformation from proliferation to differentiation in the absence of Rbpj. Collectively, these results suggest that the defective luminal closure is due to an enhanced uterine estrogenic response, which is characterized by increased epithelial proliferation and defective surface transformation.

Rbpj physically interacts with uterine ERa and suppresses hyper-estrogenic response in a Notch pathway-independent manner

To explore the potential causes underlying the enhanced uterine estrogenic response in the absence of uterine Rbpj, we examined the relationship between Rbpj and ER $\alpha$. Co-immunoprecipitation analyses revealed that Rbpj physically interacts with ER $\alpha$ both in vivo and in vitro (Figure 4D and 4E). This result prompted us to further pursue the correlation between Rbpj and estrogenic effects in Ishikawa cell line, a human endometrial cell line that expresses $E R \alpha$ and responds sensitively to $E_{2}$.

Rbpj, or together with DNMAML, a dominant-negative form of MAML which blocks the Notch-dependent Rbpj funciton [40] was transfected into Ishikawa cells. Rbpj-transfected cells showed significantly suppressed estrogenic responses, as evidenced by the downregulation of ER $\alpha$ target genes (IGF1 and $c M y c$ ) [41] following $\mathrm{E}_{2}$ treatment (Figure $4 \mathrm{~F}$ ); these cells also showed decreased cell proliferation during in vitro culture after Rbpj transfection (Figure 4G). Co-transfection of DNMAML did not reverse these effects (Figure 4F and $4 \mathrm{G})$, indicating that the suppression of hyper-estrogenic response by Rbpj is exerted in a Notch pathway-independent manner. To further explore whether the interaction of Rbpj with ER $\alpha$ affects the DNA binding activity of ER $\alpha$, we performed ChIP-qPCR analysis of ER $\alpha$-binding elements on $I G F-1$ and $c M y c$ genes (Figure $4 \mathrm{H}$ and $4 \mathrm{~J}$ ) in Ishikawa cells with or without Rbpj transfection under 
A

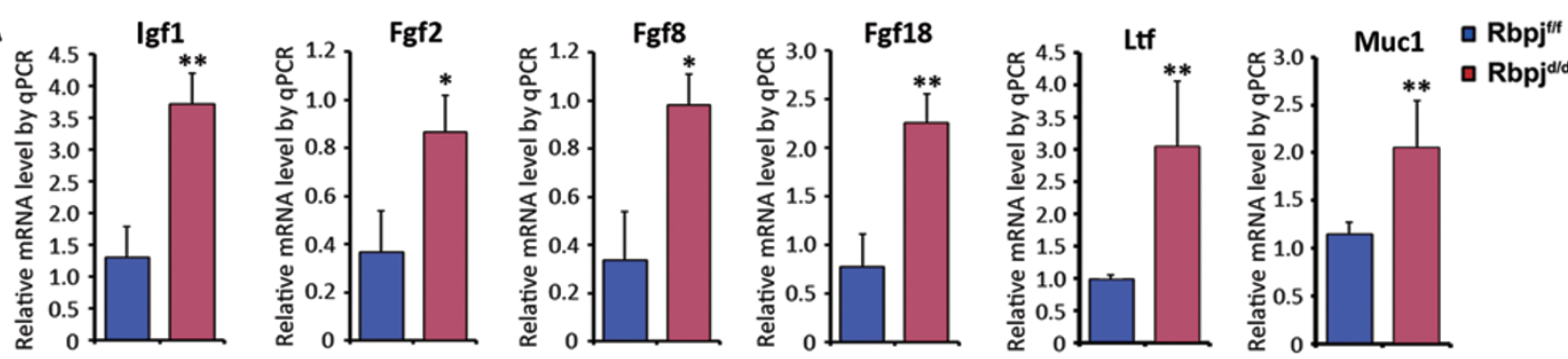

B

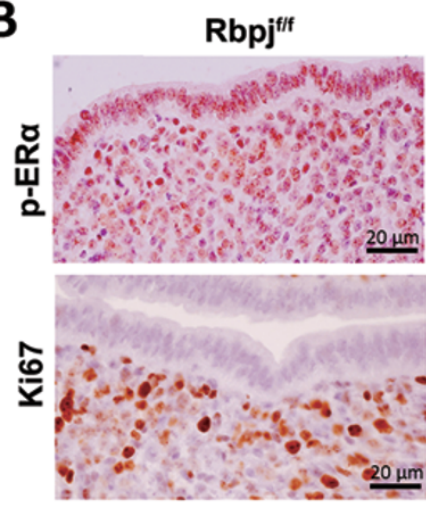

$\mathrm{Rbpj}^{\mathrm{d} / \mathrm{d}}$

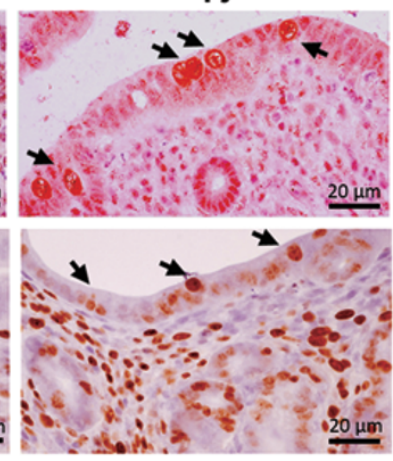

C

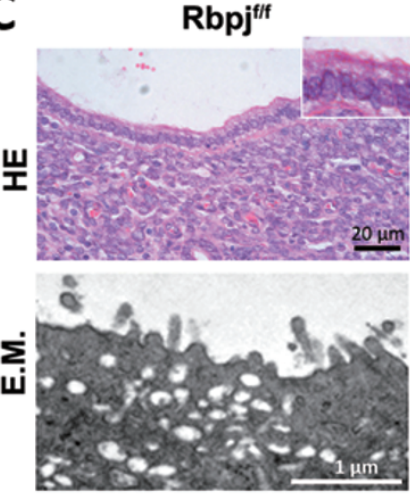

$\mathbf{R b p j}^{\mathrm{d} / \mathrm{d}}$
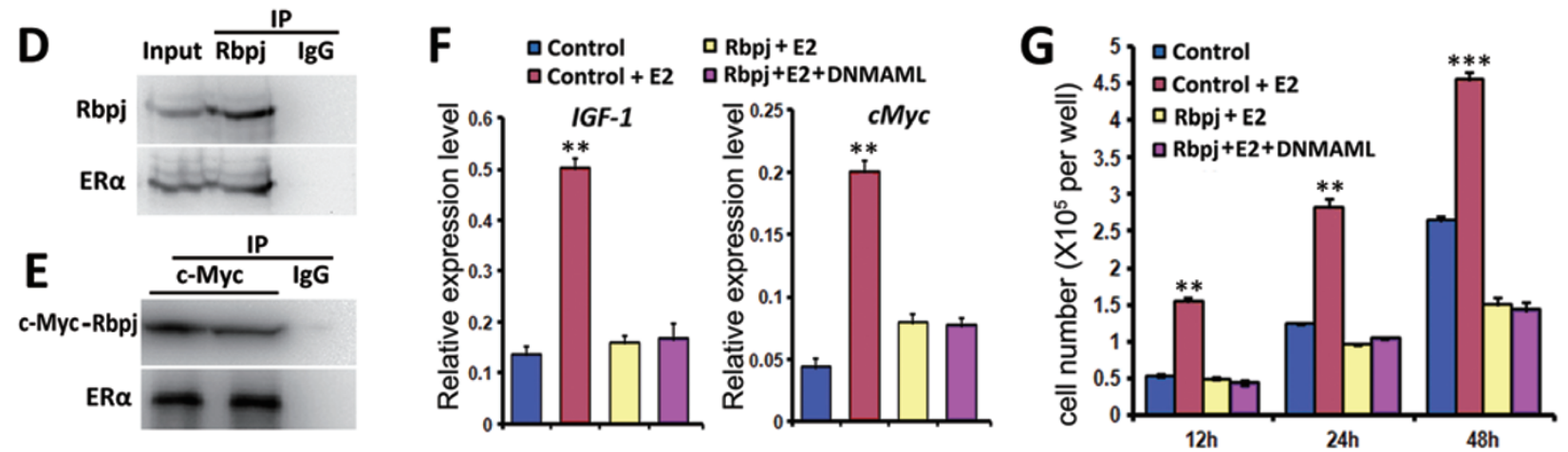

H

Human IGF-1 promoter

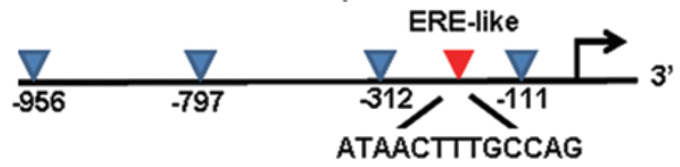

I

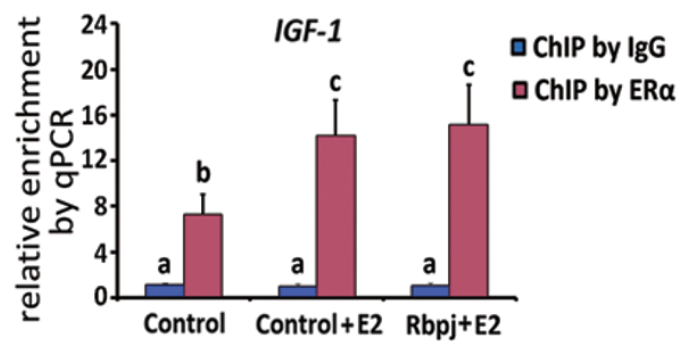

J Human cMyc enhancer
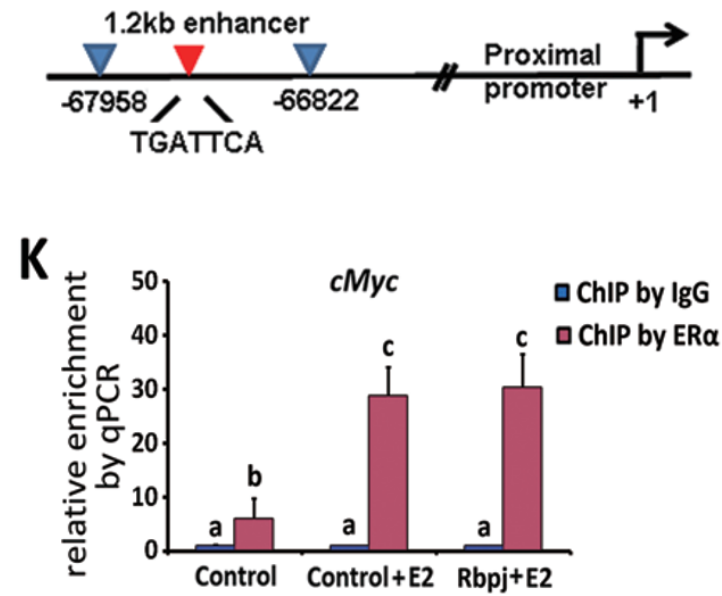
Figure $4 \mathrm{Rbpj}$ suppresses the estrogenic response by physically interacting with ER $\alpha$ in a Notch pathway-independent manner. (A) Relative expression levels of estrogen-regulated genes in $R b p j^{f / f}$ and $R b p j^{d / d}$ mice on day 4 of pregnancy. The values are normalized to the GAPDH expression level and indicated as the mean \pm SEM. $n=3$. $* P<0.05$, ** $P<0.01$. (B) Immunohistochemistry of $\mathrm{Ki} 67$ and $\mathrm{p}-\mathrm{ER} \alpha$ in $R b p j^{f / f}$ and $R b p j^{d / d}$ uteri on day 4 of pregnancy. The black arrows indicate positive signals in the uterine epithelium. (C) HE staining and electron microscopy analysis of the uterine epithelial surface of day 4 uteri. E.M., electron microscopy. (D, E) Immunoprecipitation assays for detecting the interaction of Rbpj and ER. In D, day 4 uterine tissue lysates were subjected to immunoprecipitation using an anti-Rbpj antibody or goat IgG, followed by immunoblotting with anti-Rbpj and anti-ER $\alpha$ antibodies. In E, Ishikawa cell lysates (after transfection with c-Myc-tagged Rbpj vectors) were subjected to immunoprecipitation using an anti-c-Myc antibody or goat IgG, followed by immunoblotting with anti-c-Myc and anti-ER $\alpha$ antibodies. (F) $\mathrm{E}_{2}$-regulated genes IGF1 and cMyc were measured by quantitative RT-PCR for transfected Ishikawa cells. Results are normalized to the level of GAPDH and indicated as the mean \pm SEM. $n=3 . * * P<0.01$. (G) Measurements of cell proliferation by MTS assay after $12 \mathrm{~h}, 24 \mathrm{~h}$, and $48 \mathrm{~h}$ transfection. The values represent the mean \pm SEM of six replicates from three independent experiments. $* * P<0.01, * * * P<0.01$. (H, I) Schematic representation of promoter region for IGF1 with ERE site indicated by a red arrowhead (H); ChIP-qPCR result showed enriched binding of ER $\alpha$ to the ERE site (I). (J, K) Schematic representation of promoter region for $c M y c$ with ERE site indicated by a red arrowhead (J); ChIP-qPCR result showed enriched binding of ER $\alpha$ to the ERE site (K). In I and $\mathbf{K}$, different letters represent statistical significance (a vs $b$ and a vs c: $P<0.01$; b vs c: $P<0.05)$.

E2 treatment. As shown in Figure 4I and 4K, the expression of Rbpj did not affect the binding affinity of ER $\alpha$ on the target genes, suggesting that the suppression effects of Rbpj on ER $\alpha$ activity involve other layers of regulation, such as affecting protein-protein interactions.

Defective post-implantation uterine patterning is associated with abnormal vasculature remodeling and decreased MMP expression

The histological examination performed on days 6-8 revealed an abnormal decidual shape in the $R b p j^{d / d}$ uteri (Figure 2A and 2E), suggesting that this defect is related to aberrant decidual remodeling at post-implantation stages. Indeed, by using alkaline phosphatase (ALP) staining, which is a conventional method to reveal the decidualizing stroma [26], we found that the $R b p j^{d / d}$ uteri showed an abnormal pattern characterized by diffused ALP staining and the lack of clear polarization that is normally observed in $R b p j$ fff mice (Figure 5A). The expression of Vegf, which is important for stimulating vasculogenesis and angiogenesis, also showed an abnormal pattern (Supplementary information, Figure S7). Besides the abnormal uterine shape and decidual pattern, we also found that vasculature within decidual bed is abnormal in $\mathrm{Rbpj}^{d / d}$ mice. Immunostaining of CD31, an endothelial marker, showed a disorganized pattern coupled with significantly decreased intensity around the implanting embryo (Figure 5A). It is likely that the abnormal vasculature system in Rbpj mutant uteri would fail to provide sufficient nutrient supply to the growing embryo, which might be a major cause for the compromised embryo development.

The pattern of extracellular laminin deposition is another hallmark of remodeling of uterine decidua [42]. By employing immunofluorescence staining, we found that the laminin organization within the mesometrial zone was also different in the $R b p j^{d / d}$ uteri; the organization was marked by the presence of only small, round sinuses that failed to elongate as in $\mathrm{Rbpj}^{\mathrm{fff}}$ uteri (Supplementary information, Figure S7C and S7D). As previous studies have reported that inhibition of uterine proteinases also resulted in defects in post-implantation decidual patterning and vasculature remodeling $[42,43]$ similar to the phenotype of Rbpj mutant mice, we next examined the expression of Mmp2 and $M m p 9$, the two major proteinases expressed in decidualizing uteri and developing conceptus. Quantitative PCR and in situ hybridization experiments both revealed that the expression of $M m p 2$ and $M m p 9$ was significantly downregulated in $R b p j{ }^{d / d}$ uteri during days 6-8 compared with $R_{b p j} j^{\text {fff }}$ uteri (Figure $5 B, 5 C$ and Supplementary information, Figure S8). Additionally, overall activities of MMP in post-implantation uteri were significantly decreased in $\mathrm{Rbpj}^{d / d}$ females (Figure 5D).

Rbpj transcriptionally regulates Mmp2 gene expression in a Notch pathway-dependent manner

As $M m p 2$ is primarily expressed in the decidual bed and overlapped with $R b p j$, we further explored the potential mechanisms by which Rbpj regulates $M m p 2$ expression through ChIP analysis. The DNA fragments that were immunoprecipitated with the anti-Rbpj antibody were PCR-amplified with eight pairs of primers flanking the potential Rbpj-binding sequence [44] within the regulatory region of Mmp2 (Figure 5E). The results showed that with the P5 ( $-101 \mathrm{~F} / \mathrm{R}$ primers) primers, an amplicon was detected from the input DNA and from DNA fragments that were immunoprecipitated by the anti-Rbpj antibody, indicating that Rbpj binds to the -101 site of the $M m p 2$ promoter (Figure 5F). To further confirm the 

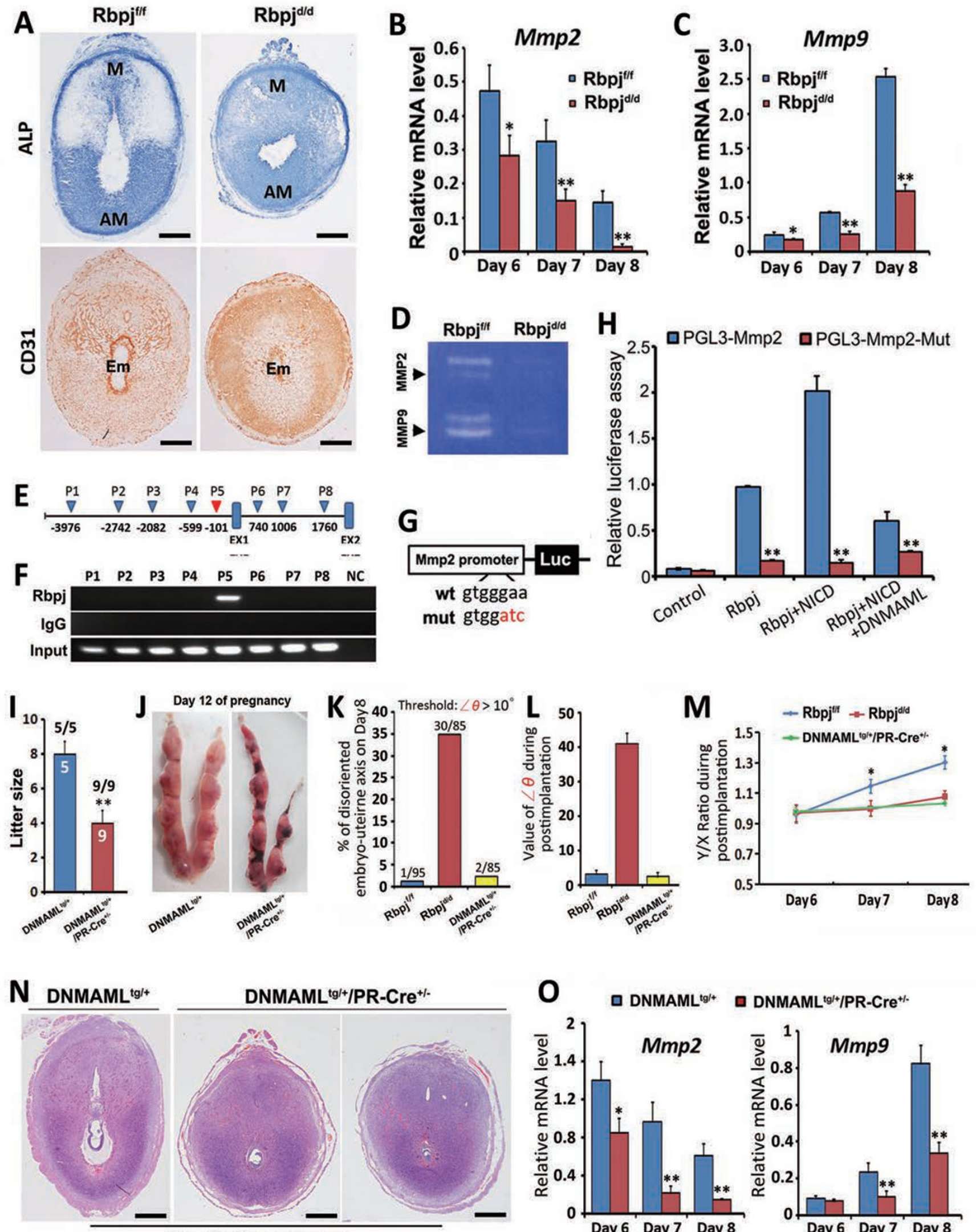

Day 8 of pregnancy

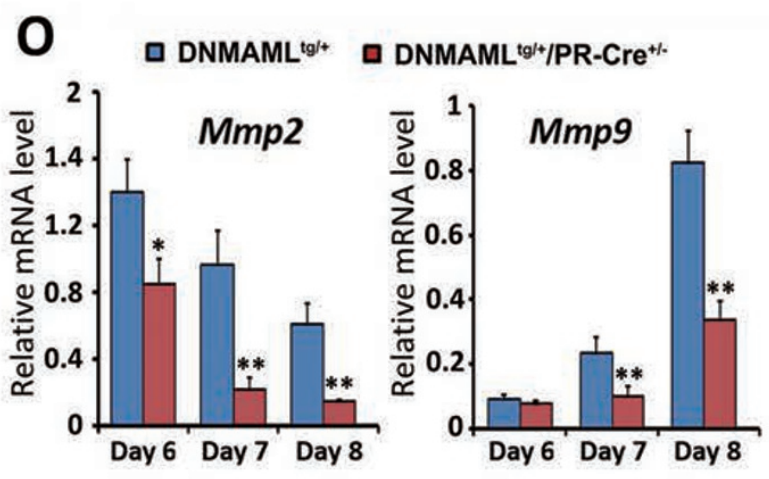


interaction between Rbpj and the Mmp2 promoter, a dual-luciferase assay using reporter constructs containing the P5 locus of the Mmp2 promoter was performed (Figure $5 \mathrm{G})$. Rbpj, or together with NICD or with both NICD and DNMAML were transfected into 293T cells. The transfection of $R b p j$ or together with NICD significantly enhanced luciferase activity, while co-expression of DNMAML efficiently reversed the effects (Figure $5 \mathrm{H}$ ), demonstrating that $\mathrm{Mmp} 2$ transcription is directly regulated by Rbpj through the canonical Notch pathway.

DNMAML ${ }^{\text {tg+ }}$ mouse mimics the phenotype of Rbpj deletion in decidual remodeling, but not embryonic-uterine disorientation

To further confirm the scenario of Rbpj function regarding Notch pathway-independent and -dependent mechanisms, we crossed DNMAML-transgenic ( $D N$ $\left.M A M L^{\text {tg+}}\right)$ mice, in which $D N M A M L$ gene is knocked into the ROSA26 locus [45], with $P R-\mathrm{Cre}^{+/-}$mice, generating $D N M A M L^{\text {tg+ }} / P R-\mathrm{Cre}^{+/-}$mice with DNMAML specifically overexpressed in the mouse uterus. We observed that $D N M A M L^{t g^{+}} / P R-\mathrm{Cre}^{+/-}$females showed reduced fertility, but not did not reach the severity of Rbpj mutant mice (Figure 5I and 5J). Further examination of DNMAML ${ }^{t g^{+}} /$ $P R-\mathrm{Cre}^{+/-}$pregnant mice at post-implantation stages revealed that these mice showed a phenotype of abnormal decidual patterning and decreased expression of MMPs, similar to RBPJ mutant mice (Figure 5M-5O), but the embryonic-uterine orientation was not disrupted (Figure $5 \mathrm{~K}$ and $5 \mathrm{~L}$ ). These in vivo data well-recapitulate the con- clusion that Rbpj-mediated uterine functions include both Notch pathway-independent mechanism that governs embryonic-uterine orientation and Notch pathway-dependent mechanism that regulates decidual remodeling.

Pharmacologically tuning down ERa activity corrected embryonic-uterine disorientation, but not defective decidual remodeling

Given that the abnormal luminal closure and resulting embryonic-uterine disorientation are due to enhanced uterine estrogenic response in $R b p j^{d / d}$ uteri, we further tested whether the suppression of the estrogenic response by pharmacologically antagonizing ER $\alpha$ could rescue the defective luminal closure in $R b p j j^{d / d}$ uteri. We treated $R_{b p j} f / f$ and $R b p j^{d / d}$ mice with a low dose of ER antagonist (ICI 182,780) at midnight on day 3 , which represents a timing prior to pre-implantation $\mathrm{E}_{2}$ secretion. The ICI treatment strikingly reduced the expression of $\mathrm{E}_{2}$ target genes on day 4 (Figure 6A). Additionally, the abnormal expression of the p-ER $\alpha$ and Ki67 was largely corrected following the ER antagonist treatment (Figure 6B). Most importantly, the abnormal luminal closure process was corrected in the $\mathrm{Rbpj}^{d / d}$ mice following the ICI treatment, and the blastocyst was located at the normal implantation chamber with M-AM orientation on day 5 (Figure 6C), and the fertility of $\mathrm{Rbpj}^{d / d}$ mice after ICI treatment showed a significant improvement regarding pregnancy rate and average litter size (Figure 6D-6F). By judging the histology of day 8 implantation sites after ICI treatment, although the orientation problem has been rescued,

Figure 5 Uterine deletion of $R b p j$ affects uterine decidual patterning by directly regulating $M m p 2$ in a Notch pathway-dependent manner. (A) ALP staining and CD31 immunohistochemistry of day 8 implantation sites in $R b p j^{f / f}$ and $R b p j^{d / d}$ mice. Note the diffused staining without clear polarization in the $R b p j^{d / d}$ uteri, and disorganized vasculature compared with $R b p j^{f / f}$ uteri. Scale bar, $500 \mu \mathrm{m}$. (B, C) Quantitative RT-PCR analysis revealed the decreased expression levels of $M m p 2$ (B) and Mmp9 (C) mRNAs in $R_{b p j} f^{f / f}$ and $R b p j^{i / d}$ implantation sites on days 6-8 of pregnancy. The values are normalized to the GAPDH expression level and are indicated as the mean \pm SEM. $n=3$. $* P<0.05$, ** $P<0.01$. (D) Representative zymographic analysis of MMP activities from $R b p j^{f / f}$ and $R b p j^{d / d}$ day 8 implantation sites. Similar results were obtained in three independent experiments. (E) Schematic representation of the genomic structure of Mmp2. The triangles indicate potential Rbpj-binding elements within a region spanning $5 \mathrm{~kb}$ upstream of transcription starting site and intron-1. EX, Exon. (F) ChIP assay showing enriched binding of Rbpj to the Mmp2 promoter region. The locations of eight primer sets (P1 to P8) were designed to detect the Rbpj-binding sites. The P5 region, which is highlighted in red in $\mathbf{E}$, represents an enriched binding region for Rbpj in the Mmp2 gene. (G) Schematic representation of the luc-Mmp2 and luc-Mmp2-mut reporter constructs for the enhancer assays. (H) Cis-activation potential of the region containing P5 or its mutant evaluated by luciferase assays. The values are shown as the mean \pm SEM. ${ }^{* *} P<0.01 . n=3$. (I) Average litter sizes in $D N M A M L^{\text {tg/t }}$ and $D N M A M L^{\text {tg/ }} / P R-\mathrm{Cre}^{+-}$mice. ${ }^{* *} P<0.01$. Numbers above bars represent number of mice gave birth/number of plugged mice. (J) Representative uteri from $D N M A M L^{\text {tg/t }}$ and $D N M A M L^{\text {tg/t/}} / P R-\mathrm{Cre}^{+/-}$mice on pregnant day 12. (K) The percentage of uterine-embryo disorientation in day 8 implantation sites. The threshold of $\angle \theta$ analyzed is $>10^{\circ}$. (L) The value of $\angle \theta$ during post-implantation stages examined in day 8 implantation sites. $n=80-90$ implantation sites for each time point. (M) The ratio of the uterine short $(\mathrm{X})$ and long $(\mathrm{Y})$ axes in days 6-8 implantation sites, $n=80-90$ implantation sites for each time point. Data shown represent the mean $\pm \mathrm{SEM}$. $* P<0.05$. (N) Representative cross-sections of $D N M A M L^{\text {tg/ }}$ and $D N M A M L^{\text {tg/+ }} / P R-\mathrm{Cre}^{+/-}$implantation sites on day 8. Note the shortened $Y$ axis in $D N M A M L^{t g / t} / P R-C r e^{+/-}$mice. Scale bar, $500 \mu \mathrm{m}$. (0) Quantitative RT-PCR analysis revealed the decreased expression levels of Mmp2 and Mmp9 mRNAs in the DNMAML $L^{\text {tg/t }}$ and DNMAML $L^{\text {tg/t}} / P R-C r e^{+/-}$implantation sites on days 6-8 of pregnancy. Data shown represent the mean \pm SEM. $n=3$. $* P<0.05, * * P<0.01$. 
A $\square \mathrm{Rbpj}^{\mathrm{fff}} \square \mathrm{Rbpj}^{\mathrm{d} / \mathrm{d}} \square \mathrm{Rbpj} \mathrm{j}^{\mathrm{d} / \mathrm{d}}+\mathrm{ICl}$
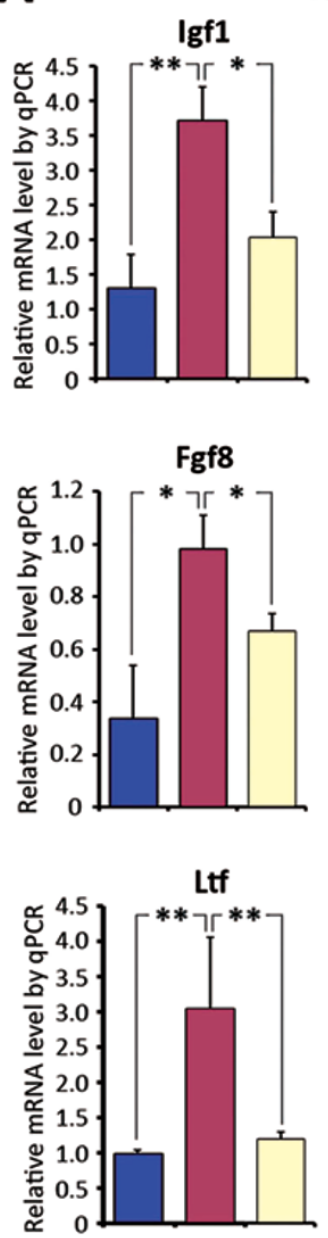

Fgf2
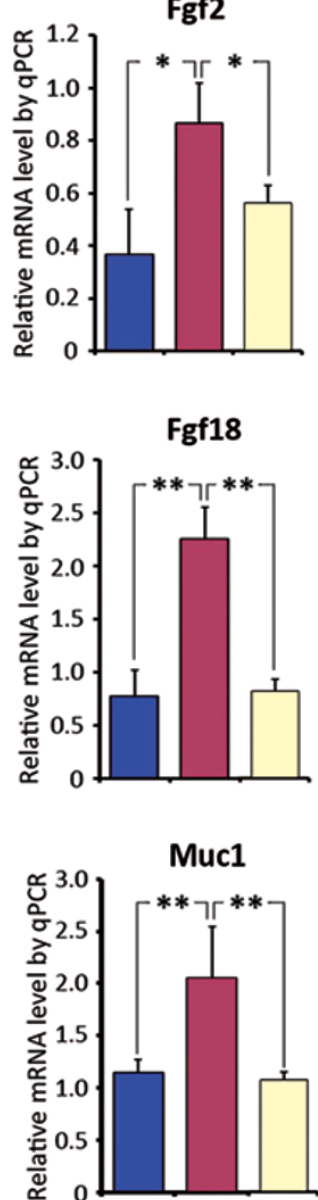

B Rbpjald, vehicle
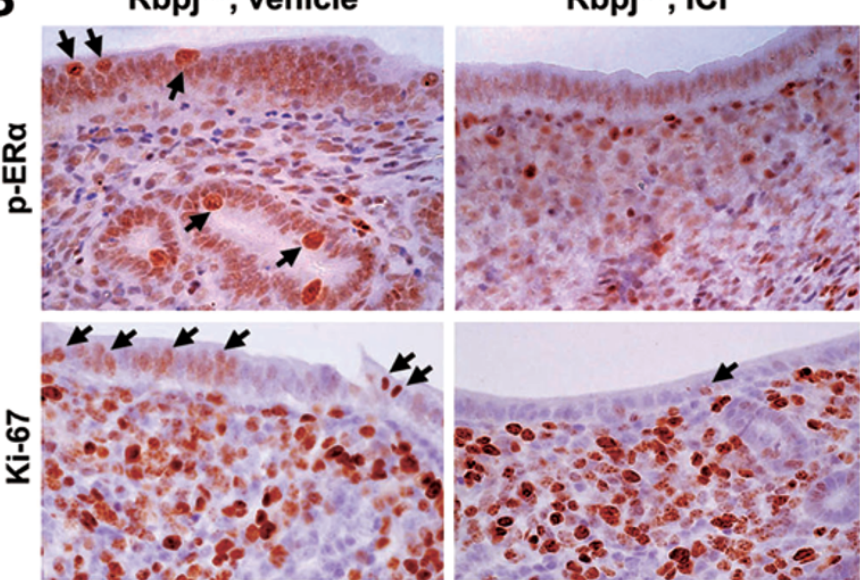

C

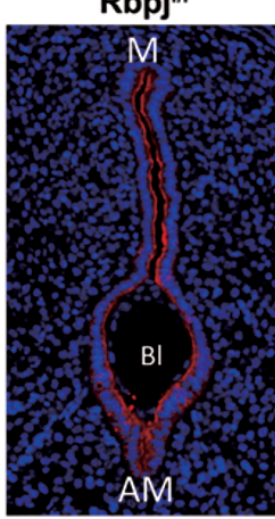

$\mathbf{R b p j}^{\mathrm{d} / \mathrm{d}}$, vehicle $\quad \mathbf{R b p j}^{\mathrm{d} / \mathrm{d}}, \mathrm{ICl}$

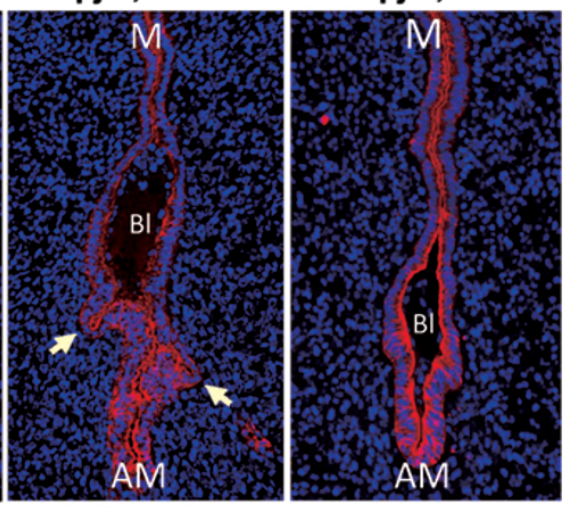

CK/Hochest
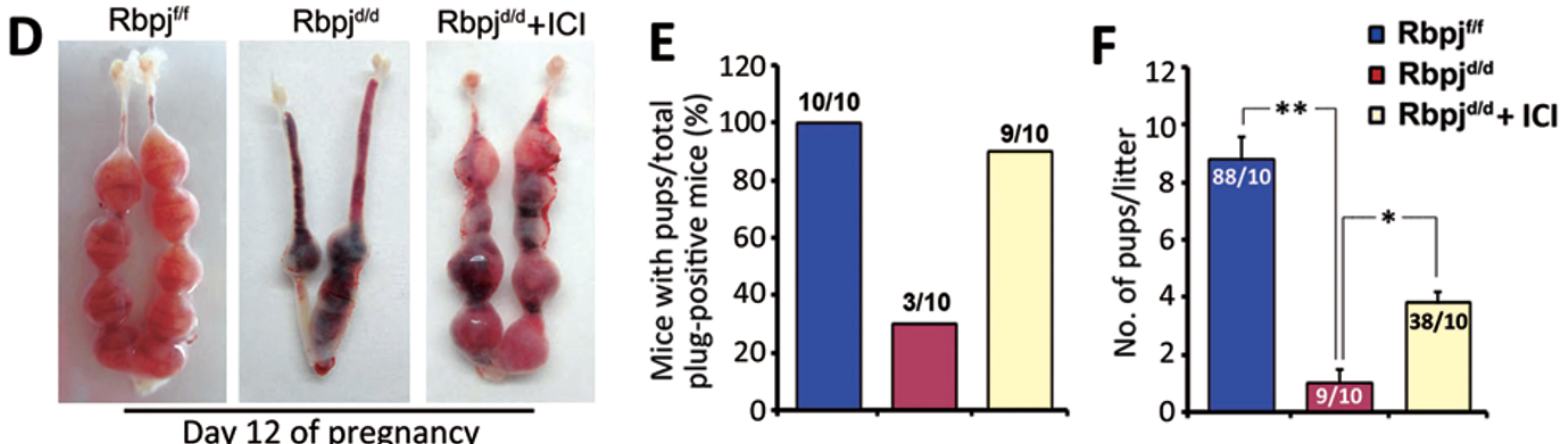

Figure 6 Inhibition of $\mathrm{E}_{2}$ hyper-sensitivity with $\mathrm{ICI}$ treatment rescues defective luminal closure and partially restores $R b p j{ }^{d / d}$ mouse fertility. (A) Relative expression levels of $\mathrm{E}_{2}$-regulated genes in day 4 uteri in $R b p j^{f / f}$ and $R b p j^{\mathrm{d} / d}$ mice treated with a sesame oil vehicle or low-dose ICI. ICI, ICI 182780. The values are normalized to the GAPDH expression level and indicated as the mean \pm SEM. $n=3$. ${ }^{*} P<0.05,{ }^{*} P<0.01$. (B) Immunohistochemical staining of $\mathrm{p}$-ER $\alpha$ and Ki-67 in vehicle- or ICl-treated $R b p j / d$ mice on day 4. The black arrows indicate positive signals. (C) Representative photos of immunofluorescence staining of untreated $\mathrm{Rbpj}^{\mathrm{f} / \mathrm{f}}$, and vehicle- or ICl-treated $\mathrm{Rbpj}^{\mathrm{d} / \mathrm{d}}$ implantation sites on day 5. Similar results were obtained in $6 \mathrm{ICl}$-treated $R b p j^{d / d}$ mice. The white arrows denote extra epithelial folds. Cy3-labeled cytokeratin is shown in red; Hoechst 33342-labeled nuclei are shown in blue. (D) Representative uteri from $R b p j^{f / f}, R b p j^{d / d}$ and ICl-treated $R b p j^{i / d}$ females on day 12 . (E) Pregnancy outcomes in $R b p j^{f / f}, R b p j^{d / d}$ and ICl-treated $R b p j^{d / d}$ females. (F) Average litter sizes in $R b p j^{f / f}, R b p j^{d / d}$ and ICl-treated $R b p j^{d / d}$ females, $* P<0.05,{ }^{* *} P<0.01$. Numbers within bars indicate the number of implantation sites/number of mice examined. 
the abnormal decidual patterning ( $\mathrm{Y} / \mathrm{X}$ ratio) remained (Supplementary information, Figure S9), thus reinforcing the conclusion that Rbpj functions in pregnant uteri are stage-specific with different regulatory mechanisms.

\section{Discussion}

The molecular mechanisms that control mammalian embryo orientation are enigmatic. The present study showed a surprising phenotype that uterine deletion of Rbpj signaling results in a progressively disorientated uterine-embryonic axis at post-implantation stages, which stems from the defective luminal closure before embryo-uterine attachment that caused by uterine hyper-estrogenic response. We further revealed that Rbpj physically interacts with ER $\alpha$ to ensure normal estrogenic effects within uterine context via Notch pathway-independent mechanisms. At post-implantation stages, Rbpj directly regulates uterine $M m p 2$ expression via Notch pathway-dependent mechanisms, which ensures normal decidual remodeling. The uterine deletion of Rbpj results in embryonic-uterine disorientation and abnormal decidual remodeling, which lead to restrained embryo growth and substantial embryo loss.

The process of pre-implantation uterine luminal closure, in which the randomly shaped luminal folds change to a highly regulated slit-like structure with a fixed M-AM direction, provides a directional landscape that guides proper embryo orientation at the time of initial implantation, therefore directing subsequent uterus-embryo alignments. On day 4 of pregnancy in mice, the regulation of pre-implantation luminal closure is controlled by a $\mathrm{P}_{4}$-dominated uterine environment in coordination with a small surge of pre-implantation estrogen secretion [46]. A successful luminal closure results from regulated stromal edema and nonproliferating opposing epithelia that are closely attached to one another $[12,47]$. In our study, we found that the uterine deletion of Rbpj disturbed this regulated process by unleashing ER $\alpha$ activity, which resulted in enhanced estrogenic response in the uterine context. Although the incomplete luminal closure in $R b p j j^{d / d}$ uteri was not sufficiently severe to abrogate the implantation process, it produced abnormal luminal folds that had axes that were not parallel to the uterine M-AM axis (Figure 7). When a blastocyst is faced with multiple luminal folds, the extra luminal folds exists as many "pitfalls"; once a blastocyst falls into one fold by chance, it will establish an embryonic axis that depends on the direction of the crypt that it accommodates with, and an initial misorientation (with regard to the uterine A-AM axis) will then lead to a catastrophically disoriented uterine-embryonic axis. A recent study of uterine Msxl deletion also reported defects in luminal closure that were characterized by the presence of extra uterine crypts for blastocyst attachment; the authors also reported increased pregnancy loss during mid-gestation [48]. Our current findings strongly suggest that normal establishment of embryonic-uterine orientation in mammals is not only guided by intrinsically programmed embryonic signals, but also depends on the coordinated transformation of uterine lumen as a proper guide.

Another interesting result of the present study is that although $\mathrm{Rbpj}$ is primarily expressed in the uterine stroma during the peri-implantation period, its deletion seems to cause not only an enhanced estrogenic response in the stroma but also a profound effect on the uterine epithelial layer, as evidenced by significantly increased epithelial proliferation, $\mathrm{E}_{2}$-responsive gene expression and an altered apical surface structure. This interesting phenomenon further indicates an intimate stromal-epithelial interaction during the peri-implantation period, which depends on the synergistic and antagonistic effects of $\mathrm{P}_{4}$ and $\mathrm{E}_{2}$ on uterine cell proliferation and differentiation through their receptors $[12,49]$. Emerging evidence has indicated that paracrine factors (such as IGF and FGF family members) that are generated in the stroma cells and induced by estrogen through ER receptors function as critical intermediate signaling molecules that stimulate epithelial proliferation $[37,38]$. Our data showed that uterine deletion of $R b p j$, which is localized in the stroma, results in substantial increased expression of IGFs and FGFs, which subsequently enhances estrogenic responses in the epithelia layer. To search for the causes, we further demonstrated that Rbpj can physically interact with $\mathrm{ER} \alpha$ to ensure a normal estrogen activity in uterine contexts prior to embryo-uterus attachment, which is Notch pathway-independent. The detailed mechanism by which Rbpj suppresses ER $\alpha$ function in uterine context is currently unclear and remains an interesting topic that warrants further investigation.

MMPs are the most prominent family of proteinases associated with multiple physiological and pathological processes $[50,51]$. Previous studies have indicated that at post-implantation stages, the normal process of decidual remodeling into an egg-shaped uterine pattern depends on developmentally expressed MMP genes, as their abrogation would lead to abnormal uterine shape remodeling characterized by a shortened length of the uterine long-axis and a less-strictly regulated embryo orientation $[42,43,52]$. These data suggest that normal uterine stromal remodeling is synchronized with the process of the formation of a proper uterine-embryonic axis and at least plays a role in optimizing this process [8]. Additionally, because Mmp2 is dynamically expressed in the uterine context [52], its dynamic expression pattern should be regulated by upstream transcription factors that are de- 


\section{Normal mice uterine Rbpj deletion}

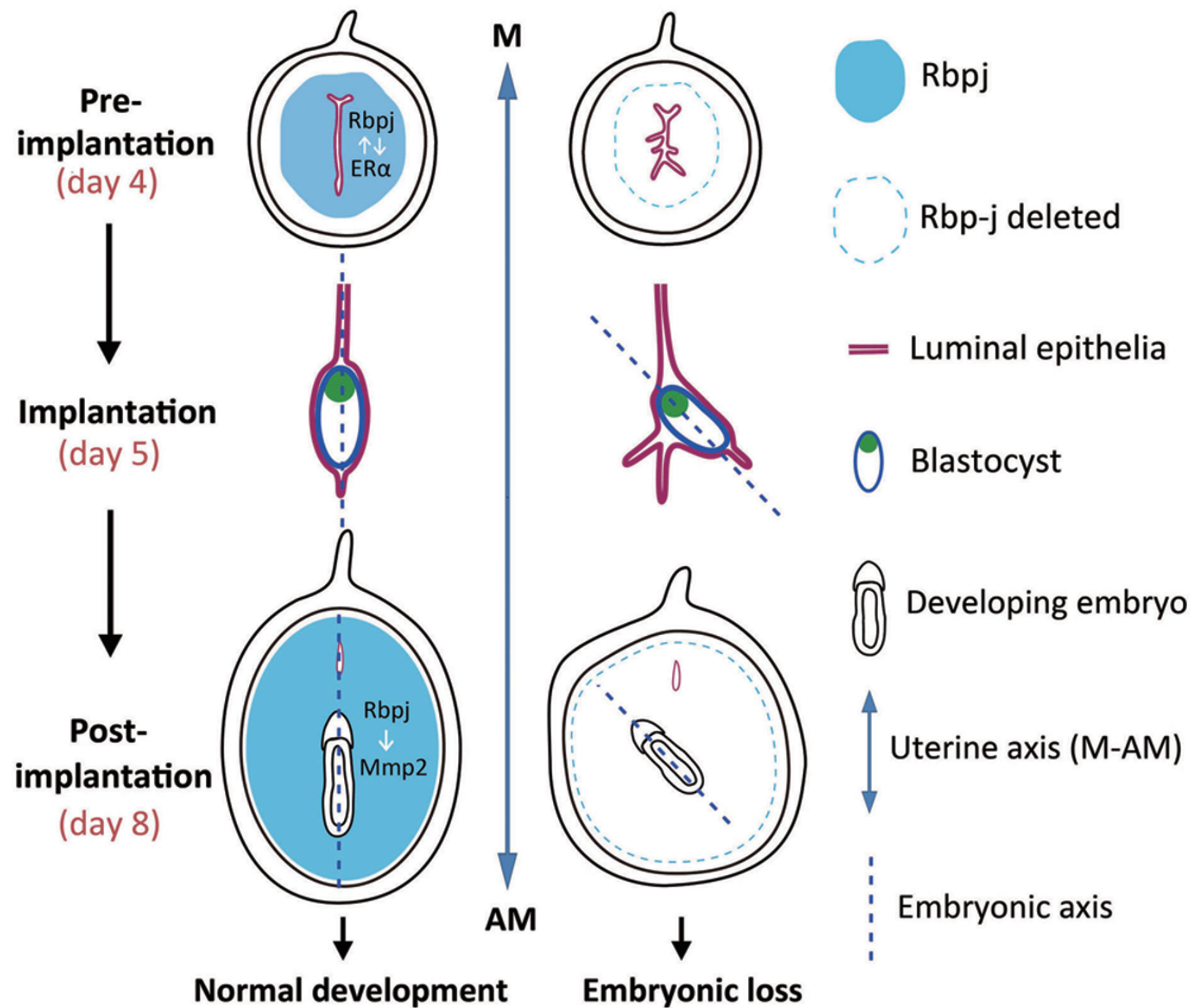

Figure 7 Illustrative model of uterine Rbpj's role during the peri-implantation period for embryo development. In normal situation (left), the embryonic axis is consistent with the uterine M-AM axis. In uteri containing a specific deletion of $R b p j$ (right), the process of uterine luminal closure is defective during the pre-implantation period, leading to the formation of extra epithelial folds that "trap" the implanting blastocyst, which is caused by unleashed ER $\alpha$ activity due to the loss of physical interaction of Rbpj and ER $\alpha$ (Notch pathway-independent). After the blastocyst establishes the embryonic axis in an abnormal uterine fold, the development of the embryonic axis will deflect from the uterine axis. During post-implantation development, the loss of Rbpj results in abnormal decidual patterning due to decreased Mmp2 expression that is transcriptionally regulated by Rbpj (Notch pathway-dependent). The combined defects in uterine-embryonic orientation and decidual patterning in $R b p j^{d / d}$ mice lead to subsequent embryonic lethality.

velopmentally regulated by the progression of implantation and decidualization. In the present study, we demonstrated that in Rbpj mutant uteri, there are decreased expression and enzymatic activity of MMPs, and we further revealed that Rbpj directly binds to the promoter region of Mmp2 in post-implantation uteri and promotes its transcriptional activity in a Notchpathway-dependent manner. The decreased activities of MMPs are supposed to exaggerate the progressively disoriented uterine-embryonic axis as previous studies have shown that when there is an aberrant decidual growth, uterine M-AM orientation is usually deviated with regards to polarized gene expression $[13,14,53]$, supporting a hypothesis that deferential decidual patterning could help optimizing the uterine-embryo orientation during post-implantation stages development. In the present study, it is also no- 
table that while Rbpj is deleted in the uterine part, the trophoblastic MMP9 from embryonic part also seems to be downregulated (Supplementary information, Figure S8), which is most probably a result of retarded embryo growth due to either the misoriented embryonic axis or the abnormal decidual vasculature, or both. In such case, the reduced trophoblastic MMP9 expression further limits the ability of trophoblast invasion and vascular remodeling, creating a situation that the defects from both decidual and embryonic part become mutually reinforcing, which results in a vicious circle that finally leads to the death of embryo in the Rbpj mutant uteri. Notably, similar scenario might also exist in a recently published paper showing that both maternal and embryonic MMP9 are important for normal placenta formation at fetal-maternal interface and thus for embryo development [54], recapitulating our point that the development of embryo is inextricably linked to the uterine signaling, which, in this case, is governed by uterine Rbpj.

In summary, we provide the first genetic evidence that uterine Rbpj, functioning through both Notch pathway-independent and -dependent mechanisms, is essential for instructing the initial uterine-embryonic orientation and ensuring normal decidual patterning for normal embryo development, which, in our belief, substantiates a novel concept that normal mammalian embryonic-uterine orientation requires proper guidance from developmentally controlled uterine signaling.

\section{Materials and Methods}

\section{Animals and treatments}

$\mathrm{Rbpj}^{\text {loxp/loxp }}$ mice were generated as previously described [25] and kindly provided by Dr Tasuku Honjo. DNMAML ${ }^{t g /+}$ mice were generated as previously described [45]. Uterine-specific mutant mice were generated by crossing $R b p j^{\text {loxp/loxp }}$ or $D N M A M L^{\operatorname{tg} /+}$ mice with $P R-\mathrm{Cre}^{+/-}$mice [27]. All mice were housed in the Institutional Animal Care Facility according to institutional guidelines for laboratory animals. Female mice were mated with fertile WT males to induce pregnancy (vaginal plug $=$ day 1 of pregnancy). To examine implantation, pregnant dams were killed on day 5 (0900 h). Implantation sites were visualized by an intravenous injection of Chicago blue dye solution, and the number of implantation sites, demarcated by distinct blue bands, was recorded. For ICI treatment, each mouse was intraperitoneally injected with $10 \mathrm{ng}$ in $200 \mu \mathrm{l}$ of sesame oil at $10 \mathrm{pm}$ on day 3. Implantation sites were analyzed on day 5 .

\section{Immunostaining}

Immunohistochemistry was performed in 5 - $\mu \mathrm{m}$-thick paraffin-embedded sections using specific antibodies as indicated. Tissues were fixed in 10\% neutral buffered formalin. Microwave heating was used for antigen retrieval. For immunohistochemistry analysis, antibodies specific to ER $\alpha$ (Dako, 1:100), p-ER $\alpha$ (Santa Cruz Biotechnology, 1:100), progesterone receptor (PR) (DAKO,
1:100), Ki-67 (Epitomics, 1:200), COX2 (Santa Cruz Biotechnology, 1:200), CD31 (BD, 1:50) were used in 5- $\mu \mathrm{m}$ thick paraffin-embedded sections. A Histostain-SP Kit (Zhongshan Golden Bridge Biotechnology) was used to visualize the antigen. For immunofluorescence, double-immunofluorescence staining for SMA (Bio Genex, 1:100) and cytokeratin (DAKO, 1:100) was performed in paraffin-fixed sections and secondary antibodies conjugated with $\mathrm{Cy} 2$ and $\mathrm{Cy} 3$ dyes were used, respectively. Immunofluorescence for Laminin (Sigma, 1:200) and cytokeratin was performed in fresh-frozen sections. Nuclear staining was performed using Hochest 33343 (Sigma, $0.1 \mu \mathrm{g} / \mathrm{ml}$ ). All frozen sections were fixed in $10 \%$ neutral buffered formalin and incubated with primary antibody at $4{ }^{\circ} \mathrm{C}$ overnight, followed by incubation with secondary antibody at room temperature for $1 \mathrm{~h}$. Immunofluorescence images were captured in a Zeiss LSM 510 confocal scanning laser microscope.

\section{In situ hybridization}

In situ hybridization with isotopes or digoxygenin (DIG) was performed as previously described [55]. Mouse-specific cRNA probes for Rbpj, Bmp2, IL11R $\alpha, W n t 4, M m p 2$ and Mmp9 were used for hybridization. Cryosections hybridized with sense probes served as negative controls. Whole mount in situ hybridization of Cerl1 on isolated embryos was performed as previously described [56].

\section{Co-immunoprecipitation}

Decidual cells were isolated as described previously [57]. Nuclear protein was extracted by using nuclear extraction kit according to the manufacturer's instructions (Millipore). Protein lysates $(1 \mathrm{mg})$ from decidual cells or Ishikawa cells transfected with c-Myc-tagged Rbpj plasmid were incubated with $4 \mu \mathrm{g}$ of anti-Rbpj antibody (Santa Cruz Biotechnology) or anti-c-Myc antibody (ABclonal) and rotated overnight at $4{ }^{\circ} \mathrm{C}$. Protein Aagarose beads (Thermo) were washed and incubated with protein lysates overnight at $4^{\circ} \mathrm{C}$. Immunoprecipitated proteins were separated by SDSPAGE and detected by immunoblotting using antibody specific to $\mathrm{ER} \alpha$ (Dako), Rbpj or c-Myc. The control immunoprecipitation was performed by incubating the lysates with goat IgG (Zhongshan Golden Bridge Biotechnology).

\section{Real-time RT-PCR analysis}

RT-PCR was performed as described [58]. Total RNA was isolated from uterine tissues or cells with Trizol reagent following the manufacturer's protocol. A total of 1-3 $\mu \mathrm{g}$ RNA was used to synthesize cDNA. The expression levels of different genes were validated by RT-PCR analysis using the ABI 7500 sequence detector system according to manufacturer's instructions (Applied Biosystems). All primers for real-time PCR were listed in Supplementary information, Table S1. Assays were performed at least three times with each in duplicate.

\section{Western blotting}

Protein extraction and western blotting were performed as previously described [55]. Antibodies to Rbpj and actin (Sigma) were used. Actin served as a loading control.

\section{Serum $P_{4}$ and $E_{2}$ level measurement}

Mouse blood samples were collected on day 4 (0900 h). Serum 
$\mathrm{P}_{4}$ and $\mathrm{E}_{2}$ levels were measured by radioimmunoassay (RIA).

\section{ChIP analysis}

ChIP analyses were performed as previously described [59]. The binding of Rbpj to Mmp2 was assessed by ChIP assay using decidual tissues of day 6 implantation sites. The binding of ER $\alpha$ to $I G F-1$ and $c M y c$ was assessed by ChIP assay using Ishikawa cell line. The ERE sites on Igf-1 and cMyc were previously described $[60,61]$. In brief, the ChIP assay was performed according to the instructions of ChIP assay Kit (Millipore) with slight modifications. On day 6 of pregnancy, the decidua was isolated from the uterus and cut into small pieces (3-4 $\mathrm{mm}$ in length). Tissues were then suspended in $1 \%$ formaldehyde (Sigma)-PBS solution for $10 \mathrm{~min}$ at room temperature. The crosslinking was terminated by adding glycine to a final concentration $0.125 \mathrm{M}$. Tissues were then collected by centrifugation at $5000 \times \mathrm{g}$ for $5 \mathrm{~min}$ at $4{ }^{\circ} \mathrm{C}$ and washed twice with ice-cold PBS. Cell pellets were lysed in $1 \%$ SDS buffer containing a protease inhibitor. Chromatin was sheared by sonication until the average length of DNA was $~ 500$ bp as evaluated by agarose gel electrophoresis. The sheared chromatin was diluted in ChIP dilution buffer to a final SDS concentration of $0.1 \%$. Salmon sperm DNA/protein Aagarose slurry was added to preclear the chromatin solution. $1 \%$ of the chromatin fragments were stored at $-20{ }^{\circ} \mathrm{C}$ to be used later for non-precipitated total chromatin (input) for normalization. 99\% of chromatin fragments were incubated with $4 \mu \mathrm{g}$ anti-Rbpj antibody overnight at $4{ }^{\circ} \mathrm{C}$. Normal goat IgG (Santa Cruz Biotechnology) was used as a negative control for nonspecific immunoprecipitation. The chromatin-antibody complex was incubated with protein $\mathrm{A} / \mathrm{G}$ beads for 1 $\mathrm{h}$ at $4{ }^{\circ} \mathrm{C}$. The antibody/DNA complex on the agarose beads was then collected by centrifugation. The beads were washed in the order of low salt immune complex buffer, high salt immune complex buffer, $\mathrm{LiCl}$ immune complex buffer, and TE buffer provided by the supplier. The beads were suspended in elution buffer and the precipitated protein/DNA complexes were eluted from the antibodies/beads. The resulting protein/DNA complexes were subjected to cross-link reversal in $5 \mathrm{M} \mathrm{NaCl}$ at $65^{\circ} \mathrm{C}$ for $4 \mathrm{~h}$ followed by the addition of $0.5 \mathrm{M}$ EDTA, $1 \mathrm{M}$ Tris- $\mathrm{HCl}$, and $10 \mu \mathrm{g} / \mathrm{ml}$ proteinase $\mathrm{K}$ (Sigma) at $45^{\circ} \mathrm{C}$ for $1 \mathrm{~h}$. DNA was purified by phenol/chloroform extraction and ethanol precipitation. Specific primers were used to detect immunoprecipitated chromatin fragments, as well as input chromatin (Supplementary information, Table S1).

\section{Zymography}

Decidual cells were isolated from $R b p j^{f f f}$ and $R b p j^{d / d}$ females on day 8 of pregnancy. Lysates of decidual fractions were prepared by homogenizing tissue pieces in substrate gel sample buffer for $10 \mathrm{~min}$ at $37^{\circ} \mathrm{C}$. Zymography and reverse zymography were performed as previously described [62].

\section{Cell transfection, MTS and luciferase assay}

Ishikawa cells were transfected with plasmids using lipofectamine 2000 (Invitrogen) according to the manufacturer's instructions. The Ishikawa cells were maintained at $37{ }^{\circ} \mathrm{C}$ in an atmosphere of $5 \% \mathrm{CO}_{2} / 95 \%$ air in DMEM medium supplemented with $10 \%(\mathrm{v} / \mathrm{v})$ FBS. Cells were plated in six-well plates and transfected with plasmid DNA (with amounts shown in each figure legend, brought up to $2 \mu \mathrm{g}$ in total with empty vectors) using Lipofectamine 2000 the next day at a cell confluence of about
$80 \%$. Treatment with $0.1 \%(\mathrm{v} / \mathrm{v})$ DMSO (vehicle) or $100 \mathrm{nM} \mathrm{E}$ in medium was performed $12 \mathrm{~h}$ after transfection and cells were collected after another $12 \mathrm{~h}$ for RNA and protein extraction. For proliferation study, cells were seeded in 96-well plates at a density of $5 \times 10^{4}$ cells/well and processed following plasmid transfection procedures. Treatment with $\mathrm{E}_{2}$ in medium was performed for the indicated times as described for each experiment. Cell proliferation was measured as a function of 3-(4, 5-dimethylthiazolyl-2)-2, 5-diphenyltetrazolium bromide oxidation (MTS Cell Proliferation Assay Kit) according to the manufacturer's instructions. Experiments were performed on three separate occasions.

For luciferase assay, the upstream region of the $M m p 2$ gene relative to the transcription start site was generated by PCR (Sense: 5'-AGAGATCTA GGAGCTCAGCGTCCTGATTC-3'; anti-sense: 5'-ATAAGCTTGGCTCAGGAG CTCATAACGGG-3') using uterus genomic DNA as template. The amplified fragments were cloned into pGL3-Basic vectors (Promega). Mutations of the Rbpj-binding sites were achieved by Fast Mutagenesis System (Stratagene). HEK293T cells were kept in DMEM medium plus $10 \%$ FBS. All constructs were transiently transfected into $293 \mathrm{~T}$ cells using Lipofectamine 2000 according to the manufacturer's instructions. pRL-TK, internal control plasmid expressing Renilla (Promega) was co-transfected into the cells to normalize firefly luciferase activity of the reporter plasmids. Cells were collected 48 $\mathrm{h}$ after transfection of reporter plasmids and luciferase activities were measured using the Dual-Luciferase Reporter Assay System. Assays were performed at least three times with each in duplicate.

\section{Statistical analysis}

Statistical analysis was performed with SPSS11.5 program. Comparison of means was performed using student's $t$-test. Data were shown as means \pm SEM.

\section{Acknowledgments}

We are grateful to Dr Tasuku Honjo (Kyoto University) for providing us the $R b p j^{f / f}$ mice. We thank Dr Sudhansu K Dey (University of Cincinnati College of Medicine) for proofreading the manuscript. This work was supported by the National Basic Research Program of China (973 Program; 2011CB944400 to HBW, HL and YW), the National Natural Science Foundation of China (81130009 and 81330017 to HBW, 31200879 to QC, and 31300957 to YZ) and the Strategic Priority Research Program of the Chinese Academy of Sciences (XDA04020202-20 and XDA04020419 to ED).

\section{References}

1 Assheton R. On the Causes which lead to the attachment of the mammalian embryo to the walls of the uterus. $Q J \mathrm{Mic} S \mathrm{Sc}$ 1894; 37:173-190.

2 Wimsatt WA. Some comparative aspects of implantation. Biol Reprod 1975; 12:1-40.

3 Rossant J, Tam PP. Emerging asymmetry and embryonic patterning in early mouse development. Dev Cell 2004; 7:155164.

4 Wang H, Dey SK. Roadmap to embryo implantation: clues from mouse models. Nat Rev Genet 2006; 7:185-199.

5 Smith LJ. Embryonic axis orientation in the mouse and its 
correlation with blastocyst relationships to the uterus. Part 1 . Relationships between 82 hours and 4 1/4 days. J Embryol Exp Morphol 1980; 55:257-277.

6 Smith LJ. Embryonic axis orientation in the mouse and its correlation with blastocyst relationships to the uterus. II. Relationships from 4 1/4 to 9 1/2 days. J Embryol Exp Morphol 1985; 89:15-35.

7 Cha J, Sun X, Dey SK. Mechanisms of implantation: strategies for successful pregnancy. Nat Med 2012; 18:1754-1767.

8 Chen Q, Zhang Y, Elad D, et al. Navigating the site for embryo implantation: Biomechanical and molecular regulation of intrauterine embryo distribution. Mol Aspects Med 2013; 34:1024-1042.

9 Mesnard D, Filipe M, Belo JA, Zernicka-Goetz M. The anterior-posterior axis emerges respecting the morphology of the mouse embryo that changes and aligns with the uterus before gastrulation. Curr Biol 2004; 14:184-196.

10 Weber RJ, Pedersen RA, Wianny F, Evans MJ, Zernicka-Goetz M. Polarity of the mouse embryo is anticipated before implantation. Development 1999; 126:5591-5598.

11 Tranguch S, Cheung-Flynn J, Daikoku T, et al. Cochaperone immunophilin FKBP52 is critical to uterine receptivity for embryo implantation. Proc Natl Acad Sci USA 2005; 102:14326-14331.

12 Zhang S, Lin H, Kong S, et al. Physiological and molecular determinants of embryo implantation. Mol Aspects Med 2013; 34:939-980.

13 Daikoku T, Song H, Guo Y, et al. Uterine Msx-1 and Wnt4 signaling becomes aberrant in mice with the loss of leukemia inhibitory factor or Hoxa-10: evidence for a novel cytokine-homeobox-Wnt signaling in implantation. Mol Endocrinol 2004; 18:1238-1250.

14 Paria BC, Ma W, Tan J, et al. Cellular and molecular responses of the uterus to embryo implantation can be elicited by locally applied growth factors. Proc Natl Acad Sci USA 2001; 98:1047-1052.

15 Bray SJ. Notch signalling: a simple pathway becomes complex. Nat Rev Mol Cell Biol 2006; 7:678-689.

16 Guruharsha KG, Kankel MW, Artavanis-Tsakonas S. The Notch signalling system: recent insights into the complexity of a conserved pathway. Nat Rev Genet 2012; 13:654-666.

17 Johnson JE, Macdonald RJ. Notch-independent functions of CSL. Curr Top Dev Biol 2011; 97:55-74.

18 Hori K, Cholewa-Waclaw J, Nakada Y, et al. A nonclassical bHLH Rbpj transcription factor complex is required for specification of GABAergic neurons independent of Notch signaling. Genes Dev 2008; 22:166-178.

19 Kato Y. The multiple roles of Notch signaling during left-right patterning. Cell Mol Life Sci 2011; 68:2555-2567.

20 Krebs LT, Iwai N, Nonaka S, et al. Notch signaling regulates left-right asymmetry determination by inducing Nodal expression. Genes Dev 2003; 17:1207-1212.

21 Lopes SS, Lourenco R, Pacheco L, et al. Notch signalling regulates left-right asymmetry through ciliary length control. Development 2010; 137:3625-3632.

22 Rock JR, Gao X, Xue Y, et al. Notch-dependent differentiation of adult airway basal stem cells. Cell Stem Cell 2011; 8:639-648.

23 Tanigaki K, Tsuji M, Yamamoto N, et al. Regulation of alpha- beta/gammadelta $\mathrm{T}$ cell lineage commitment and peripheral $\mathrm{T}$ cell responses by Notch/RBP-J signaling. Immunity 2004; 20:611-622.

24 Oka C, Nakano T, Wakeham A, et al. Disruption of the mouse RBP-J kappa gene results in early embryonic death. Development 1995; 121:3291-3301.

25 Han $\mathrm{H}$, Tanigaki K, Yamamoto $\mathrm{N}$, et al. Inducible gene knockout of transcription factor recombination signal binding protein-J reveals its essential role in T versus B lineage decision. Int Immunol 2002; 14:637-645.

26 Lee K, Jeong J, Kwak I, et al. Indian hedgehog is a major mediator of progesterone signaling in the mouse uterus. Nat Genet 2006; 38:1204-1209.

27 Soyal SM, Mukherjee A, Lee KY, et al. Cre-mediated recombination in cell lineages that express the progesterone receptor. Genesis 2005; 41:58-66.

28 Bilinski P, Roopenian D, Gossler A. Maternal IL-11Ralpha function is required for normal decidua and fetoplacental development in mice. Genes Dev 1998; 12:2234-2243.

29 Franco HL, Dai D, Lee KY, et al. WNT4 is a key regulator of normal postnatal uterine development and progesterone signaling during embryo implantation and decidualization in the mouse. FASEB J 2011; 25:1176-1187.

30 Kurihara I, Lee DK, Petit FG, et al. COUP-TFII mediates progesterone regulation of uterine implantation by controlling ER activity. PLoS Genet 2007; 3:e102.

31 Li Q, Kannan A, Wang W, et al. Bone morphogenetic protein 2 functions via a conserved signaling pathway involving Wnt4 to regulate uterine decidualization in the mouse and the human. J Biol Chem 2007; 282:31725-31732.

32 Morris SA, Grewal S, Barrios F, et al. Dynamics of anterior-posterior axis formation in the developing mouse embryo. Nat Commun 2012; 3:673.

33 Yamamoto M, Saijoh Y, Perea-Gomez A, et al. Nodal antagonists regulate formation of the anteroposterior axis of the mouse embryo. Nature 2004; 428:387-392.

34 Lim H, Paria BC, Das SK, et al. Multiple female reproductive failures in cyclooxygenase 2-deficient mice. Cell 1997; 91:197-208.

35 Sun X, Zhang L, Xie H, et al. Kruppel-like factor 5 (KLF5) is critical for conferring uterine receptivity to implantation. Proc Natl Acad Sci USA 2012; 109:1145-1150.

36 Xie H, Wang H, Tranguch S, et al. Maternal heparin-binding-EGF deficiency limits pregnancy success in mice. Proc Natl Acad Sci USA 2007; 104:18315-18320.

37 Li Q, Kannan A, DeMayo FJ, et al. The antiproliferative action of progesterone in uterine epithelium is mediated by Hand2. Science 2011; 331:912-916.

38 Zhu L, Pollard JW. Estradiol-17beta regulates mouse uterine epithelial cell proliferation through insulin-like growth factor 1 signaling. Proc Natl Acad Sci USA 2007; 104 (40):1584715851.

39 Kawagoe J, Li Q, Mussi P, et al. Nuclear receptor coactivator-6 attenuates uterine estrogen sensitivity to permit embryo implantation. Dev Cell 2012; 23:858-865.

40 Weng AP, Nam Y, Wolfe MS, et al. Growth suppression of pre-T acute lymphoblastic leukemia cells by inhibition of notch signaling. Mol Cell Biol 2003; 23:655-664.

41 Shah YM, Rowan BG. The Src kinase pathway promotes 
tamoxifen agonist action in Ishikawa endometrial cells through phosphorylation-dependent stabilization of estrogen receptor (alpha) promoter interaction and elevated steroid receptor coactivator 1 activity. Mol Endocrinol 2005; 19:732748.

42 Afonso S, Romagnano L, Babiarz B. The expression and function of cystatin $\mathrm{C}$ and cathepsin $\mathrm{B}$ and cathepsin $\mathrm{L}$ during mouse embryo implantation and placentation. Development 1997; 124:3415-3425.

43 Alexander CM, Hansell EJ, Behrendtsen O, et al. Expression and function of matrix metalloproteinases and their inhibitors at the maternal-embryonic boundary during mouse embryo implantation. Development 1996; 122:1723-1736.

44 Mumm JS, Kopan R. Notch signaling: from the outside in. Dev Biol 2000; 228:151-165.

$45 \mathrm{Tu} \mathrm{L}$, Fang TC, Artis D, et al. Notch signaling is an important regulator of type 2 immunity. $J$ Exp Med 2005; 202:10371042.

46 Dey SK, Lim H, Das SK, et al. Molecular cues to implantation. Endocr Rev 2004; 25:341-373.

47 Tranguch S, Smith DF, Dey SK. Progesterone receptor requires a co-chaperone for signalling in uterine biology and implantation. Reprod Biomed Online 2006; 13:651-660.

48 Daikoku T, Cha J, Sun X, et al. Conditional deletion of Msx homeobox genes in the uterus inhibits blastocyst implantation by altering uterine receptivity. Dev Cell 2011; 21:1014-1025.

49 Cooke PS, Buchanan DL, Young P, et al. Stromal estrogen receptors mediate mitogenic effects of estradiol on uterine epithelium. Proc Natl Acad Sci USA 1997; 94:6535-6540.

50 Kessenbrock K, Plaks V, Werb Z. Matrix metalloproteinases: regulators of the tumor microenvironment. Cell 2010; 141:5267.

51 Sbardella D, Fasciglione GF, Gioia M, et al. Human matrix metalloproteinases: an ubiquitarian class of enzymes involved in several pathological processes. Mol Aspects Med 2012; 33:119-208.

52 Das SK, Yano S, Wang J, et al. Expression of matrix metalloproteinases and tissue inhibitors of metalloproteinases in the mouse uterus during the peri-implantation period. Dev Genet 1997; 21:44-54.

53 Rahman MA, Li M, Li P, et al. Hoxa-10 deficiency alters region-specific gene expression and perturbs differentiation of natural killer cells during decidualization. Dev Biol 2006; 290:105-117.

54 Plaks V, Rinkenberger J, Dai J, et al. Matrix metalloproteinase-9 deficiency phenocopies features of preeclampsia and intrauterine growth restriction. Proc Natl Acad Sci USA 2013; 110:11109-11114.

55 Lu J, Zhang S, Nakano H, et al. A positive feedback loop involving Gcm1 and Fzd5 directs chorionic branching morphogenesis in the placenta. PLoS Biol 2013; 11:e1001536.

56 Morris SA, Grewal S, Barrios F, et al. Dynamics of anterior-posterior axis formation in the developing mouse embryo. Nat Commun 2012; 3:673.

57 Hirota Y, Daikoku T, Tranguch S, et al. Uterine-specific p53 deficiency confers premature uterine senescence and promotes preterm birth in mice. J Clin Invest 2010; 120:803-815.

58 Chen Q, Zhang Y, Peng H, et al. Transient \{beta\}2-adrenoceptor activation confers pregnancy loss by disrupting embryo spacing at implantation. J Biol Chem 2011; 286:4349-4356.

59 Shi M, Hu ZL, Zheng MH, et al. Notch-Rbpj signaling is required for the development of noradrenergic neurons in the mouse locus coeruleus. J Cell Sci 2012; 125(Part 18):43204332.

60 Kang HJ, Yi YW, Kim HJ, et al. BRCA1 negatively regulates IGF-1 expression through an estrogen-responsive element-like site. Cell Death Dis 2012; 3:e336.

61 Wang C, Mayer JA, Mazumdar A, et al. Estrogen induces c-myc gene expression via an upstream enhancer activated by the estrogen receptor and the AP-1 transcription factor. $\mathrm{Mol}$ Endocrinol 2011; 25:1527-1538.

62 Kuang H, Chen Q, Zhang Y, et al. The cytokine gene CXCL14 restricts human trophoblast cell invasion by suppressing gelatinase activity. Endocrinology 2009; 150:55965605.

(Supplementary information is linked to the online version of the paper on the Cell Research website.)

(c) (i) (3) $\odot$ This work is licensed under the Creative Commons Attribution-NonCommercial-No Derivative Works 3.0 Unported License. To view a copy of this license, visit http:// creativecommons.org/licenses/by-nc-nd/3.0 